\title{
Lone axes in outer space
}

\author{
LEE MOSHER \\ CATHERINE PFAFF
}

\begin{abstract}
Handel and Mosher define the axis bundle for a fully irreducible outer automorphism in [Mem. Amer. Math. Soc. 1004 (2011)]. We give a necessary and sufficient condition for the axis bundle to consist of a unique periodic fold line. As a consequence, we give a setting, and means for identifying in this setting, when two elements of an outer automorphism group $\operatorname{Out}\left(F_{r}\right)$ have conjugate powers.
\end{abstract}

20F65; 05E18, 57M07

\section{Introduction}

We let $\operatorname{Out}\left(F_{r}\right)$ denote the outer automorphism group for a rank- $r$ free group $F_{r}$. Culler and Vogtmann [7] defined a topological space $\mathrm{CV}_{r}$, outer space, on which $\operatorname{Out}\left(F_{r}\right)$ acts properly with finite stabilizers, in analogy with the action of each mapping class group on its Teichmüller space (see Fathi, Laudenbach and Poenaru [8]). In fact, the action of each $\operatorname{Out}\left(F_{r}\right)$ on its outer space $\mathrm{CV}_{r}$ has indeed proved to possess many of the same characteristics as the action of a mapping class group on its Teichmüller space. For example, Levitt and Lustig [18] proved that, as with a pseudo-Anosov acting on Teichmüller space, each "fully irreducible" $\varphi \in \operatorname{Out}\left(F_{r}\right)$ acts with northsouth dynamics on the natural compactification $\overline{\mathrm{CV}_{r}}$ of $\mathrm{CV}_{r}$. A fully irreducible outer automorphism is the most commonly used analogue to a pseudo-Anosov. An element $\varphi \in \operatorname{Out}\left(F_{r}\right)$ is fully irreducible if no positive power $\varphi^{k}$ fixes the conjugacy class of a proper free factor of $F_{r}$.

Recall that points of outer space can be described as marked metric graphs up to isometry, by which we mean graphs whose fundamental group has been identified with the free group in a basepoint-free manner and who have lengths assigned to their edges (generally assumed to sum to one). As in Handel and Mosher [14], one can call a point $\Gamma$ in outer space a train track for $\varphi$ when there exists an affine train track representative $g: \Gamma \rightarrow \Gamma$. An affine train track representative is a train track representative, in the sense of Bestvina and Handel [4], such that each open interval inside each edge is stretched by a constant factor equal to the dilatation of $\varphi$. In [14] Handel and Mosher answered the question, posed by Vogtmann, "Is the set of train 
tracks for an irreducible automorphism contractible?" They do so by defining, for a nongeometric fully irreducible $\varphi \in \operatorname{Out}\left(F_{r}\right)$, its axis bundle, which they also show is a closed subset $\mathcal{A}_{\varphi}$ in $\mathrm{CV}_{r}$ proper homotopy equivalent to a line, invariant under $\varphi$, and such that the two ends of $\mathcal{A}_{\varphi}$ limit on the repeller and attractor of the source-sink action of $\varphi$ on $\overline{\mathrm{CV}_{r}}$. Outer automorphisms induced by homeomorphisms of compact surfaces are called geometric, and are usually primarily studied as surface homeomorphisms. If $\varphi$ is a nongeometric, fully irreducible outer automorphism, then [14, Theorem 1.1] gives three equivalent definitions of the axis bundle (see Section 2.6), the third of which is $\mathcal{A}_{\varphi}=\overline{\bigcup_{k=1}^{\infty} \mathrm{TT}\left(\varphi^{k}\right)}$, where $\operatorname{TT}\left(\varphi^{k}\right)$ is just the set of train track graphs for $\varphi^{k}$.

Unlike in the situation of a loxodromic isometry acting on hyperbolic space or of a pseudo-Anosov mapping class acting on Teichmüller space, it appears that there is in general no natural axis for a fully irreducible outer automorphism acting on outer space and that the axis bundle is a good natural analogue, in spite of in general being so far from a single axis as to actually be multidimensional. Handel and Mosher, via a list of questions in [14], and Bridson and Vogtmann [5, Question 3], more directly, ask:

Question 1.1 Describe the geometry of the axis bundle for a fully irreducible outer automorphism acting on outer space.

What we accomplish in this paper is to determine when a fully irreducible outer automorphism behaves more like a pseudo-Anosov mapping class by having an axis bundle that is just a single axis. Not only does this give a partial solution to the conjugacy problem for outer automorphisms of free groups, but it allows one to read off from an axis all train track representatives for the automorphism. Section 4 is dedicated entirely to explaining several applications of our main theorem.

The condition we prove for a unique axis relies on the ideal Whitehead graph of [14]. The condition also relies on the rotationless version $i(\varphi)$ of the index defined by Gaboriau, Jaeger, Levitt and Lustig [10] for a fully irreducible $\varphi \in \operatorname{Out}\left(F_{r}\right)$. One can think of the rotationless index as a sum of terms, each of which records the number of vertices in a component of the ideal Whitehead graph. As originally defined, the rotationless index also records the branching behavior of the attracting tree, $T_{\varphi}^{+}$, for the source-sink action of $\varphi$ on $\overline{\mathrm{CV}_{r}}$. Unlike in the surface case where one has the Poincaré-Hopf index equality, Gaboriau, Jaeger, Levitt and Lustig proved in [10] that there is instead a rotationless index inequality $0>i(\varphi) \geq 1-r$ that each fully irreducible $\varphi \in \operatorname{Out}\left(F_{r}\right)$ satisfies. (Here we have rewritten the inequality using the definition of the rotationless index given by Pfaff [21], revised to be invariant under taking powers and to have its sign be consistent with the mapping class group case.)

The notion of an ageometric fully irreducible is defined in Section 2.9. Ageometrics are believed to be generic (see, for example, Kapovich and Pfaff [16]). What we prove in 
Theorem 4.7 is a necessary and sufficient condition for an ageometric fully irreducible outer automorphism to have a unique axis. Examples of fully irreducibles satisfying the conditions of Theorem 4.7 can be found in Pfaff $[20 ; 21]$ and it was in fact proved later, in [16], that satisfying these conditions is generic along a particular "train track directed" random walk.

Theorem 4.7 The axis bundle of an ageometric, fully irreducible outer automorphism $\varphi \in \operatorname{Out}\left(F_{r}\right)$ is a unique axis precisely if both of the following two conditions hold:

(1) the rotationless index satisfies $i(\varphi)=\frac{3}{2}-r$, and

(2) no component of the ideal Whitehead graph $\operatorname{IW}(\varphi)$ has a cut vertex.

The rotationless index is always a negative half-integer. Thus, one may observe that $\frac{3}{2}-r$ is as close to equaling the bound of $1-r$ as possible without actually equaling it. As equality is achieved precisely in the case of geometric and parageometric outer automorphisms, this means that $\frac{3}{2}-r$ is the bounding ageometric rotationless index. Given a nongeometric, fully irreducible $\varphi \in \operatorname{Out}\left(F_{r}\right)$, we let $\operatorname{ST}(\varphi)$ denote the set of train track graphs for $\varphi$ on which there exists a fully stable train track representative for $\varphi$, meaning that each power is stable in the sense of [4]. We then define the stable axis bundle as $\mathcal{S A}_{\varphi}=\overline{\bigcup_{k=1}^{\infty} \mathrm{ST}\left(\varphi^{k}\right)}$; see Section 3. The stable axis bundle was introduced in [14, Section 6.5] as an object of interest. Our approach to proving Theorem 4.7 involves a study of the stable axis bundle, as proposed in [14].

Theorem 4.6 Suppose $\varphi \in \operatorname{Out}\left(F_{r}\right)$ is ageometric and fully irreducible. Then the stable axis bundle $\mathcal{S A}_{\varphi}$ is a unique axis if and only if the rotationless index satisfies $i(\varphi)=\frac{3}{2}-r$. In that case it is a unique periodic fold line.

The connection between Theorems 4.6 and 4.7 is that, for an ageometric, fully irreducible $\varphi \in \operatorname{Out}\left(F_{r}\right)$ with rotationless index $i(\varphi)=\frac{3}{2}-r$, the ideal Whitehead graph $\operatorname{IW}(\varphi)$ not having cut vertices is equivalent to the stable axis bundle in fact being the entire axis bundle. We exploit here constructions of [14, Lemma 3.1] where cut vertices lead to periodic Nielsen paths in unstable representatives.

\subsection{Remarks and further questions}

The proof of the main theorem will exhibit a sufficient condition for the axis bundle to be of dimension two or higher, namely the existence of an affine train track representative $g: \Gamma \rightarrow \Gamma$ having two or more illegal turns. In fact the axis bundle has local dimension two or higher at the point represented by $\Gamma$; see the proof of Lemma 4.3.

This condition motivates some follow-up problems regarding the behavior of higher dimensional axis bundles: 
- Is there a formula for the local dimension of the axis bundle at a point $\Gamma$ represented by an affine train track map (or more generally at any point)?

- Is the local dimension a constant function on the axis bundle?

Regarding these questions, the proof of the main theorem gives some hints. For example, here is a lower bound for the local dimension based on the illegal turn structure: each gate of $\Gamma$ of cardinality $k$ should independently contribute $k-1$ dimensions worth of folding parameters. That lower bound only takes into account "folding" of $\Gamma$, which captures just the forward direction along the axis bundle; a better dimension count would certainly have to take "unfolding" into account, in order to capture the backward direction. Also, see Comment 4.2 following the statement of Lemma 4.1 for a hint to why the answer to the second question might be "no".

The authors would like to thank the referee for the following questions:

Question 1.2 Does there exist a similar result to Theorem 4.7 for parageometric fully irreducible outer automorphisms?

Question 1.3 Is the index condition in Lemma 4.5 necessary?

Acknowledgements Pfaff would like to thank Mosher for his inspiration, support, and ideas, as well as for remaining one of her favorite people to discuss math with. She would also like to thank Thierry Coulbois and Jerome Los for illuminating conversations and their interest in her work. Finally, she would like to thank Yael Algom-Kfir, Mladen Bestvina, Michael Handel, Ilya Kapovich, and Martin Lustig for their support and thoughtful conversations about the geometry of outer space. Finally, both authors would like to thank the referee for a very careful and thoughtful reading of the paper.

Mosher is supported by a grant from the National Science Foundation. Pfaff was supported first by the ARCHIMEDE Labex (ANR-11-LABX- 0033) and the A*MIDEX project (ANR-11-IDEX-0001-02) funded by the "Investissements d'Avenir" French government program managed by the ANR. She is secondly supported by the CRC701 grant of the DFG, supporting the projects B1 and C13 in Bielefeld.

\section{Preliminary definitions and notation}

\subsection{Train track representatives}

Definition 2.1 (marked graphs and train track representatives) Let $R_{r}$ be the $r-$ petaled rose, ie the graph with precisely $r$ edges and one vertex. Recall from [4], for 
example, that a marked graph is a connected finite graph $\Gamma$, with no valence 1 or 2 vertices, together with an isomorphism $\pi_{1}(\Gamma) \cong F_{r}$ defined via a homotopy equivalence (called the marking) $\rho: \Gamma \rightarrow R_{r}$. Marked graphs $\rho: \Gamma \rightarrow R_{r}$ and $\rho^{\prime}: \Gamma^{\prime} \rightarrow R_{r}$ are considered equivalent when there exists a homeomorphism $h: \Gamma \rightarrow \Gamma^{\prime}$ such that $\rho^{\prime} \circ h$ is homotopic to $\rho$. A homotopy equivalence $g: \Gamma \rightarrow \Gamma$ of a marked graph $\Gamma$ is a train track representative for $\varphi \in \operatorname{Out}\left(F_{r}\right)$ if it maps vertices to vertices, $\varphi=g_{*}: \pi_{1}(\Gamma) \rightarrow$ $\pi_{1}(\Gamma)$, and $\left.g^{k}\right|_{\operatorname{int}(e)}$ is locally injective for each edge $e$ of $\Gamma$ and $k>0$.

Many of the definitions and notation for discussing train track representatives were established in [4] and [3]. We recall some here.

Definition 2.2 (turns, gates and directions) Let $g: \Gamma \rightarrow \Gamma$ be a train track representative of $\varphi \in \operatorname{Out}\left(F_{r}\right)$. By a direction at a vertex $v$ we will mean a germ of initial segments of directed edges emanating from $v$. The definition can be extended to an interior point $x$ of an edge $e$ by defining a direction at $x$ to be a germ of open segments of $e$ with $x$ as a boundary point. $D g$ will denote the direction map induced by $g$.

We call a point $v$ periodic if there exists a $j \geq 1$ such that $g^{j}(v)=v$. And we call a point $v$ preperiodic if there exists a point $w$ and $j \geq 1$ such that $g^{j}(v)=g^{j}(w)=w$. The same definitions apply for a lift $\tilde{g}$ acting on $T=\widetilde{\Gamma}$. We call a direction $d$ at a periodic point $v$ periodic if $D g^{k}(d)=d$ for some $k>0$.

We call an unordered pair of directions $\left\{d_{i}, d_{j}\right\}$, based at the same point, a turn. It is an illegal turn for $g$ if $D g^{k}\left(d_{i}\right)=D g^{k}\left(d_{j}\right)$ for some $k$ and a legal turn otherwise. Considering the directions of an illegal turn equivalent, one can define an equivalence relation on the set of directions at a vertex. Each equivalence class is called a gate.

Directions and turns at a point $v$ in a simplicial tree $T$ can be analogously defined. More generally, a direction at a point $v$ in an $F_{r}$-tree $T$ can be defined as a component of $T \backslash\{v\}$. If $T$ is a train track, then, given a lift $\widetilde{g}: \widetilde{\Gamma} \rightarrow \widetilde{\Gamma}$ fixing some vertex $\widetilde{v}$, we can define the direction map $D \widetilde{g}$ at $\widetilde{v}$. A periodic direction for $\widetilde{g}$ is then a direction mapped into itself by $D \widetilde{g}^{k}$ for some $k \in \mathbb{Z}_{>0}$. The direction is fixed if $k=1$

\subsection{Periodic Nielsen paths and (fully) stable representatives}

Throughout this subsection, $\varphi \in \operatorname{Out}\left(F_{r}\right)$ is fully irreducible and $g: \Gamma \rightarrow \Gamma$ is a train track representative of $\varphi$. (Hence, in particular, $g$ is expanding and irreducible.)

Definition 2.3 (periodic Nielsen paths and principal points) We call a locally injective path tight. Recall from [4] that a nontrivial tight path $\rho$ in $\Gamma$ is called a periodic Nielsen path (PNP) for $g$ if $g^{k}(\rho) \simeq \rho$ rel endpoints for some $k$. It is called a Nielsen path (NP) 
if the period is one and an indivisible Nielsen path $(i N P)$ if it further cannot be written as a concatenation $\rho=\rho_{1} \rho_{2}$, where $\rho_{1}$ and $\rho_{2}$ are also NPs for $g$.

As in [14], we call a periodic point $v \in \Gamma$ principal if it either has at least three periodic directions or is an endpoint of a periodic Nielsen path.

Definition 2.4 (rotationless) A train track representative is called rotationless if every principal point is fixed and every periodic direction at each principal point is fixed. Note that the rotationless property puts no restrictions on the preperiodic, nonperiodic vertices. In [9, Proposition 3.24] it is shown that one can define a fully irreducible outer automorphism to be rotationless if and only if one (hence all) of its train track representatives are rotationless.

We will use the following, which tells us that rotationless powers always exist:

Proposition 2.5 [9, Corollary 4.43] For each $r \geq 2$, there exists an $R(r) \in \mathbb{N}$ such that $\varphi^{R(r)}$ is rotationless for each $\varphi \in \operatorname{Out}\left(F_{r}\right)$.

Definition 2.6 (stable train track representatives) Let $\varphi$ be a fully irreducible outer automorphism. The paper [4] gives an algorithm for finding a representative with the minimal number of Nielsen paths; such a representative is called a stable representative. As in [14], we call a stable representative $g$ of a rotationless power $\varphi^{R}$ of $\varphi$ fully stable.

Remark 2.7 It would not affect the definition of $\bigcup \mathrm{ST}\left(\varphi^{k}\right)$ if we also called a representative fully stable whose rotationless powers are fully stable, but we will generally mean a rotationless representative when we use the term "fully stable".

\subsection{Culler-Vogtmann outer space $\mathrm{CV}_{r}$ and the attracting tree $T_{+}$}

Definition 2.8 (outer space $\mathrm{CV}_{\boldsymbol{r}}$ ) A metric on a graph $\Gamma$ is the path metric determined by choosing for each edge $e$ of $\Gamma$ a length $l(e)$ and a characteristic map $j_{e}:[0, l(e)] \rightarrow e$, in the sense of $\mathrm{CW}$ complexes. A metric is determined, up to homeomorphism isotopic to the identity, by an assignment of lengths to edges.

The volume of $\Gamma$ is defined as $\operatorname{vol}(\Gamma):=\sum_{e \in E(\Gamma)} l(e)$. A point in Culler-Vogtmann outer space $\mathrm{CV}_{r}$ is an equivalence classes of metric marked graphs of volume one, under the equivalence relation of marking-preserving isometry.

There is an unprojectivized version of outer space, denoted $\widehat{\mathrm{CV}}_{r}$, where we no longer require $\operatorname{vol}(\Gamma)=1$. 
Remark 2.9 Many definitions can be given both for $\mathrm{CV}_{r}$ and $\widehat{\mathrm{CV}}_{r}$. Hence, we sometimes blur the distinction.

Lifting to the universal cover of marked graphs, one obtains an alternative definition of $\mathrm{CV}_{r}$, used to describe the compactification. Points of compactified outer space $\overline{\mathrm{CV}_{r}}=\mathrm{CV}_{r} \cup \partial \mathrm{CV}_{r}$ can be described as equivalence classes of minimal, very small $F_{r}-$ actions on $\mathbb{R}$-trees, known as " $F_{r}$-trees". The equivalence relation is $F_{r}$-equivariant homothety. Under this description of $\overline{\mathrm{CV}_{r}}$, points of $\mathrm{CV}_{r}$ itself correspond to the simplicial $F_{r}$-trees $T$ on which $F_{r}$ acts freely; up to equivalence, such trees correspond bijectively to marked graphs via the relation of universal covering.

There are multiple equivalent descriptions of the standard topology on $\mathrm{CV}_{r}$. We describe it via its (ideal) simplicial structure. For each marked graph $\rho: \Gamma \rightarrow R_{r}$ with $N$ edges, the set of metrics on $\Gamma$ gives an $(N-1)$-dimensional open simplex in $\mathrm{CV}_{r}$ :

$$
\left\{\left(l_{1}, l_{2}, \ldots, l_{N}\right) \mid l_{k}>0, \sum l_{k}=1\right\} .
$$

Where they exist, open faces of a cell can be obtained by assigning length zero to a subset of the edges or equivalently by collapsing the forest in $\Gamma$ consisting of those edges of length zero. Faces are missing where assigning length zero to edges changes the homotopy type of the graph.

The group $\operatorname{Out}\left(F_{r}\right)$ acts on $\mathrm{CV}_{r}$ from the right, where each $\varphi \in \operatorname{Out}\left(F_{r}\right)$ acts by precomposing the marking with an automorphism representing $\varphi$. Given a fully irreducible $\varphi \in \operatorname{Out}\left(F_{r}\right)$, the repeller and attractor for the action on $\overline{\mathrm{CV}_{r}}$ are elements of $\partial \mathrm{CV}_{r}$, thus $F_{r}$-trees. We denote the attracting tree in $\partial \mathrm{CV}_{r}$ by $T_{+}^{\varphi}$, or just $T_{+}$, and the repelling tree by $T_{-}^{\varphi}$, or just $T_{-}$.

Definition 2.10 (attracting tree $T_{+}^{\varphi}$ ) We recall from [10] a concrete construction of the attracting tree $T_{+}^{\varphi}$ for a fully irreducible $\varphi \in \operatorname{Out}\left(F_{r}\right)$. Let $g: \Gamma \rightarrow \Gamma$ be a train track representative of $\varphi$ and $\widetilde{\Gamma}$ the universal cover of $\Gamma$ equipped with a distance function $\tilde{d}$ lifted from $\Gamma$. The fundamental group, $F_{r}$, acts by deck transformations, hence isometries, on $\widetilde{\Gamma}$. A lift $\widetilde{g}$ of $g$ is associated to a unique automorphism $\Phi$ representing $\varphi$. In particular, for each $w \in F_{r}$ and $x \in \widetilde{\Gamma}$, we have $\Phi(w) \tilde{g}(x)=\tilde{g}(w x)$. One can define the pseudodistance $d_{\infty}$ on $\widetilde{\Gamma}$ by $\lim _{k \rightarrow+\infty} d_{k}$, where

$$
d_{k}(x, y)=\frac{d\left(\widetilde{g}^{k}(x), \tilde{g}^{k}(y)\right)}{\lambda^{k}}
$$

for each $x, y \in \widetilde{\Gamma}$, where $\lambda>1$ is the unique positive real number such that for each $x, y \in \widetilde{\Gamma}$ the limit converges, and such that for some $x, y \in \tilde{\Gamma}$ the value of the limit is nonzero. (This number $\lambda$ is equal to the Perron-Frobenius eigenvalue of the transition 
matrix of $g$.) Then $T_{+}$is the $F_{r}$-tree defined by identifying each pairs of points $x, y \in \widetilde{\Gamma}$ such that $d_{\infty}(x, y)=0$.

\subsection{The attracting lamination $\Lambda_{\varphi}$ for a fully irreducible outer automorphism}

While one can define the set of attracting laminations for any element of $\operatorname{Out}\left(F_{r}\right)$ (see [2]), we give here only a definition yielding the unique (see [3, Lemma 1.12]) attracting lamination for a fully irreducible outer automorphism.

Let $\Gamma$ be a marked graph with universal cover $\widetilde{\Gamma}$ and projection map $p: \widetilde{\Gamma} \rightarrow \Gamma$. By a line in $\widetilde{\Gamma}$ we mean the image of a proper embedding of the real line $\tilde{\lambda}: \mathbb{R} \rightarrow \tilde{\Gamma}$. We denote by $\widetilde{\mathcal{B}}(\Gamma)$ the space of lines in $\widetilde{\Gamma}$ with the compact-open topology, generated by the open sets

$$
\tilde{\mathcal{U}}(\tilde{\gamma}):=\{L \in \widetilde{\mathcal{B}}(\Gamma) \mid \tilde{\gamma} \text { is a finite subpath of } L\} .
$$

A line in $\Gamma$ is then the image of a projection $p \circ \tilde{\lambda}: \mathbb{R} \rightarrow \Gamma$ of a line $\tilde{\lambda}$ in $\tilde{\Gamma}$, where two lines are considered equivalent when they differ via precomposition by a homeomorphism of $\mathbb{R}$. We denote by $\mathcal{B}(\Gamma)$ the space of lines in $\Gamma$ with the quotient topology induced by the natural projection map from $\widetilde{\mathcal{B}}(\Gamma)$ to $\mathcal{B}(\Gamma)$. One can then define the sets

$$
\mathcal{U}(\gamma):=\{L \in \mathcal{B}(\Gamma) \mid \gamma \text { is a finite subpath of } L\},
$$

which generate the topology on $\mathcal{B}$. For a marked graph $\Gamma$, we say a line $\gamma$ in $\Gamma$ is birecurrent if every finite subpath of $\gamma$ occurs infinitely often as an unoriented subpath in each end of $\gamma$.

Definition 2.11 (attracting lamination $\Lambda_{\varphi}$ ) Fix a fully irreducible $\varphi \in \operatorname{Out}\left(F_{r}\right)$ and consider any train track representative $g: \Gamma \rightarrow \Gamma$ for $\varphi$. Given any edge $e$ in $\Gamma$, there exists a $k>0$ such that

$$
\mathcal{U}(e) \supset \mathcal{U}\left(g^{k}(e)\right) \supset \mathcal{U}\left(g^{2 k}(e)\right) \supset \cdots
$$

is a sequence of nested open sets. The attracting lamination $\Lambda_{\varphi}$ (or just $\Lambda$ ) for $\varphi$ is the set of birecurrent lines in the intersection. We often use the same notation for the total lift $\tilde{\Lambda}$ of $\Lambda$ to the universal cover. The meaning should be clear from context.

This definition of $\Lambda$ is well-defined independent of the choice of train track representative; see [2, Lemma 1.12] for a proof. Once a basepoint lift is chosen in $\widetilde{\Gamma}$, one can identify $\partial \widetilde{\Gamma}$ with the hyperbolic boundary $\partial F_{r}$ of the free group. This allows one to identify $\tilde{\Lambda}$ with a set of unordered pairs of points in $\partial F_{r}$, by lifting $\Lambda$ via the projection $\partial F_{r}=\partial \widetilde{\Gamma} \rightarrow \mathcal{B}(\Gamma)$. It follows that $\tilde{\Lambda}$ is also well-defined. 
We may also define the realization of $\Lambda$ in a general point of outer space represented by a marked graph $\Gamma^{\prime}$ with universal cover $\widetilde{\Gamma^{\prime}}$ and with a chosen basepoint in $\widetilde{\Gamma^{\prime}}$. Using the identifications $\partial \widetilde{\Gamma} \approx \partial F_{r} \approx \partial \widetilde{\Gamma^{\prime}}$, we obtain an identification $\widetilde{\mathcal{B}}(\Gamma) \approx \widetilde{\mathcal{B}}\left(\Gamma^{\prime}\right)$, which identifies $\widetilde{\Lambda} \subset \widetilde{\mathcal{B}}(\Gamma)$ with a subset of $\widetilde{\mathcal{B}}\left(\Gamma^{\prime}\right)$ which is the realization of $\tilde{\Lambda}$ in $\widetilde{\Gamma}^{\prime}$. Following with the projection $\widetilde{\mathcal{B}}\left(\Gamma^{\prime}\right) \rightarrow \mathcal{B}\left(\Gamma^{\prime}\right)$, we obtain the realization of $\tilde{\Lambda}$ in $\Gamma^{\prime}$.

\section{5 $\Lambda$-isometries and weak train tracks}

Recall that a fully irreducible $\varphi \in \operatorname{Out}\left(F_{r}\right)$ is geometric if it is represented by a homeomorphism $f: M \rightarrow M$ of a compact surface with nonempty boundary, meaning that there exists a homotopy equivalence $h: R_{r} \rightarrow M$ with homotopy inverse $\bar{h}: M \rightarrow R_{r}$, such that the homotopy equivalence $\bar{h} f h$ is homotopic to a train track representative of $\varphi$. Let $\varphi \in \operatorname{Out}\left(F_{r}\right)$ be a nongeometric fully irreducible with attracting lamination $\Lambda$.

Definition 2.12 ( $\Lambda$-isometry) For a free, simplicial $F_{r}$-tree $T$, a $\Lambda$-isometry on $T$ is an $F_{r}$-equivariant map $f_{T}: T \rightarrow T_{+}$such that, for each leaf $L$ of $\Lambda$ realized in $T$, the restriction of $f_{T}$ to $L$ is an isometry onto a bi-infinite geodesic in $T_{+}$.

Definition 2.13 (weak train track) A normalized weak train track for $\varphi$ is a free simplicial $F_{r}$-tree $T \in \widehat{\mathrm{CV}}_{r}$ on which a $\Lambda$-isometry exists. A weak train track is an element of $\mathrm{CV}_{r}$ represented by a normalized weak train track.

\subsection{The axis bundle}

Three equivalent definitions of the axis bundle $\mathcal{A}_{\varphi}$ for a nongeometric fully irreducible outer automorphism $\varphi \in \operatorname{Out}\left(F_{r}\right)$ are given in [14]. We will use all three definitions here and thus remind the reader in this subsection of each of them. We say a few words with regard to their equivalence in Section 2.7.

Definition $2.14\left(\widehat{\mathcal{A}_{\varphi}}\right)$ Fix a normalization of $T_{+}$. Then define

$\widehat{\mathcal{A}_{\varphi}}=\left\{\right.$ free simplicial $F_{r}$-trees $T \in \widehat{\mathrm{CV}}_{r} \mid$ there exists a $\Lambda$-isometry $\left.f_{T}: T \rightarrow T_{+}\right\}$. In other words, $\widehat{\mathcal{A}_{\varphi}}$ is the set of normalized weak train tracks in $\widehat{\mathrm{CV}}_{r}$.

Definition 2.15 (fold lines) A fold line in $\mathrm{CV}_{r}$ is a continuous, injective, proper function $\mathbb{R} \rightarrow \mathrm{CV}_{r}$ defined by

(1) a continuous 1-parameter family of marked graphs $t \rightarrow \Gamma_{t}$, and

(2) a family of homotopy equivalences $h_{t s}: \Gamma_{s} \rightarrow \Gamma_{t}$ defined for $s \leq t \in \mathbb{R}$, each marking-preserving, satisfying:

Train track property $h_{t s}$ is a local isometry on each edge for all $s \leq t \in \mathbb{R}$. Semiflow property $h_{u t} \circ h_{t s}=h_{u s}$ for all $s \leq t \leq u \in \mathbb{R}$, and $h_{s s}: \Gamma_{s} \rightarrow \Gamma_{s}$ is the identity for all $s \in \mathbb{R}$. 
Axis Bundle Definition I $\mathcal{A}_{\varphi}$ is the union of the images of all fold lines $\mathcal{F}: \mathbb{R} \rightarrow \mathrm{CV}_{r}$ such that $\mathcal{F}(t)$ converges in $\overline{\mathrm{CV}_{r}}$ to $T_{-}^{\varphi}$ as $t \rightarrow-\infty$ and to $T_{+}^{\varphi}$ as $t \rightarrow+\infty$.

Axis Bundle Definition II $\mathcal{A}_{\varphi}$ is the set of weak train tracks in $\mathrm{CV}_{r}$ for $\varphi$, ie $\mathcal{A}_{\varphi}$ is the image of $\widehat{\mathcal{A}_{\varphi}}$ under the projectivization of $\widehat{\mathrm{CV}_{r}}$.

Axis Bundle Definition III $\mathcal{A}_{\varphi}=\overline{\bigcup_{k=1}^{\infty} \mathrm{TT}\left(\varphi^{k}\right)}$, where $\mathrm{TT}\left(\varphi^{k}\right)$ is the set of train track graphs for $\varphi^{k}$ and the closure is taken in $\mathrm{CV}_{r}$.

Remark 2.16 By [14, Lemma 5.1], every weak train track in $\mathrm{CV}_{r}$ is represented by a unique normalized weak train track in $\widehat{C V}_{r}$; equivalently, the projection map $\widehat{\mathrm{CV}_{r}} \rightarrow \mathrm{CV}_{r}$ restricts to a bijection $\widehat{\mathcal{A}_{\varphi}} \rightarrow A_{\varphi}$. Because of this, we will take the liberty for the rest of the paper to blur the distinction between weak train tracks and normalized weak train tracks.

Some crucial properties of the axis bundle are recorded in [14, Theorem 6.1, Lemma 6.2]. We summarize a few here as Proposition 2.17. Given a point $T \in \widehat{\mathrm{CV}}_{r}$, the length of $T$ is defined to be $\operatorname{Len}(T):=\operatorname{vol}\left(T / F_{r}\right)$.

Proposition 2.17 [14] Let $\varphi \in \operatorname{Out}\left(F_{r}\right)$ be a nongeometric fully irreducible outer automorphism. Then the map Len: $\widehat{\mathcal{A}}_{\varphi} \rightarrow(0, \infty)$ is a surjective and $\varphi$-equivariant homotopy equivalence, where $\varphi$ acts on $(0, \infty)$ by multiplication by $1 / \lambda$, using the number $\lambda$ from Definition 2.10.

\subsection{Connections between the axis bundle definitions}

The equivalence of the three axis bundle definitions is proved in [14, Theorem 1.1]. We explain here briefly connections frequently used. In particular, we show one obtains from a train track representative $g: \Gamma \rightarrow \Gamma$ of a nongeometric fully irreducible $\varphi \in \operatorname{Out}\left(F_{r}\right)$ both a $\Lambda$-isometry $g_{\infty}: \widetilde{\Gamma} \rightarrow T_{+}$and a "periodic" fold line.

\subsection{1 $\Lambda$-isometries from train track maps}

Definition 2.18 $\left(g_{\infty}\right)$ Let $g: \Gamma \rightarrow \Gamma$ be a train track representative of a nongeometric fully irreducible $\varphi \in \operatorname{Out}\left(F_{r}\right)$. We return to the construction of Definition 2.10. We let $T_{k}$ denote the simplicial $F_{r}$-tree obtained from $\widetilde{\Gamma}$ by identifying $x, y \in \widetilde{\Gamma}$ with each other when $d_{k}(x, y)=0$ and then equipping the quotient graph with the metric induced by $d_{k}$. Then, for each $i$, a basepoint-preserving lift of $g$ induces a basepoint-preserving $F_{r}$-equivariant map $\widetilde{g}_{i+1, i}: T_{i} \rightarrow T_{i+1}$ restricting to an isometry on each edge. We 
obtain a direct system $\tilde{g}_{j, i}: T_{i} \rightarrow T_{j}$ defined inductively by $\tilde{g}_{j, i}=\tilde{g}_{j, j-1} \circ \widetilde{g}_{j-1, i}$. Then the $\Lambda$-isometry $g_{\infty}: \widetilde{\Gamma} \rightarrow T_{+}$is the direct limit map:

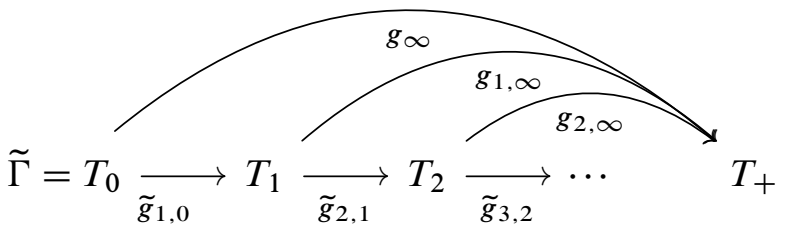

We will use the following result of Handel and Mosher.

Proposition 2.19 [14, Corollary 2.14] Let $g: \Gamma \rightarrow \Gamma$ be a train track representative of a nongeometric fully irreducible $\varphi \in \operatorname{Out}\left(F_{r}\right)$, let $\widetilde{\Gamma}$ be the universal cover, and let $[\tilde{x}, \tilde{y}]$ be the tight path from $\tilde{x}$ to $\tilde{y}$. Then $g_{\infty}: \tilde{\Gamma} \rightarrow T_{+}$is a surjective equivariant map such that, for all $\tilde{x}, \tilde{y} \in \tilde{\Gamma}$, the following are equivalent:

(1) $g_{\infty}(\tilde{x})=g_{\infty}(\tilde{y})$.

(2) There exists $k \geq 0$ such that $g_{\#}^{k}([\tilde{x}, \tilde{y}])$ is either trivial or a Nielsen path.

In particular, $g_{\infty}$ restricts to an isometry on all legal paths.

Remark 2.20 (realizing lamination leaves in $T_{+}$) Since lamination leaves are legal, Proposition 2.19 allows one to describe how lamination leaves are realized in $T_{+}$.

2.7.2 Periodic fold lines Stallings introduced "folds" in [23]. Let $g: \Gamma \rightarrow \Gamma^{\prime}$ be a homotopy equivalence of marked graphs. Let $e_{1}^{\prime} \subset e_{1}$ and $e_{2}^{\prime} \subset e_{2}$ be maximal, initial, nontrivial subsegments of edges $e_{1}$ and $e_{2}$ emanating from a common vertex and satisfying that $g\left(e_{1}^{\prime}\right)=g\left(e_{2}^{\prime}\right)$ as edge paths and that the terminal endpoints of $e_{1}^{\prime}$ and $e_{2}^{\prime}$ are in $g^{-1}(\mathcal{V}(\Gamma))$. Redefine $\Gamma$ to have vertices at the endpoints of $e_{1}^{\prime}$ and $e_{2}^{\prime}$ if necessary. One can obtain a graph $\Gamma_{1}$ by identifying the points of $e_{1}^{\prime}$ and $e_{2}^{\prime}$ that have the same image under $g$, a process we will call folding. Stallings [23] also showed that if $g: \Gamma \rightarrow \Gamma^{\prime}$ is tight, then $g$ factors as a composition of folds and a final homeomorphism. We call such a decomposition a Stallings fold decomposition. It can be obtained as follows: At an illegal turn for $g: \Gamma \rightarrow \Gamma^{\prime}$, one can fold two maximal initial segments having the same image in $\Gamma^{\prime}$ to obtain a map $\mathfrak{g}_{1}: \Gamma_{1} \rightarrow \Gamma^{\prime}$ of the quotient graph $\Gamma_{1}$. The process can be repeated for $\mathfrak{g}_{1}$ and recursively. If some $\mathfrak{g}_{k}: \Gamma_{k-1} \rightarrow \Gamma$ has no illegal turn, then $\mathfrak{g}_{k}$ will be a homeomorphism and the fold sequence is complete.

Notice that choices of illegal turns are made in this process and that different choices lead to different Stallings fold decompositions of the same homotopy equivalence. 


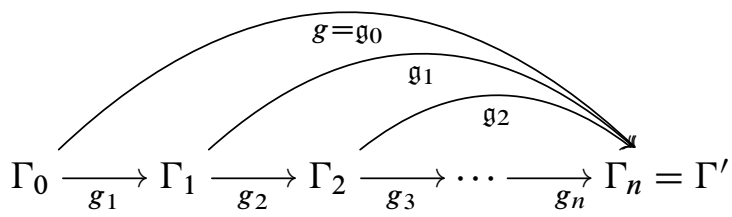

Figure 1: Constructing a Stallings folds decomposition

When $\Gamma$ is a marked metric graph (of volume 1), we obtain an induced metric on each $\Gamma_{k}$, which we may renormalize to be again of volume 1 .

In [22], Skora interpreted a Stallings fold decomposition for a tight homotopy equivalence $g: \Gamma \rightarrow \Gamma^{\prime}$ as a sequence of folds performed continuously. Repeating a Stallings fold decomposition of a train track representative for a fully irreducible outer automorphism defines a periodic fold line in outer space. The discretization of this fold line is depicted in (1), where it should be noted that $\Gamma_{n k}=\left(1 / \lambda^{n}\right) \Gamma_{0} \cdot \varphi^{n}$, for each integer $n$ :

(1) $\cdots \rightarrow \Gamma_{0} \stackrel{g_{1}}{\longrightarrow} \Gamma_{1} \stackrel{g_{2}}{\longrightarrow} \cdots \stackrel{g_{K}}{\longrightarrow} \Gamma_{K} \stackrel{g_{K+1}}{\longrightarrow} \Gamma_{K+1} \stackrel{g_{K+2}}{\longrightarrow} \cdots \stackrel{g_{2 K}}{\longrightarrow} \Gamma_{2 K} \stackrel{g_{2 K+1}}{\longrightarrow} \cdots$.

\subsection{Ideal Whitehead graphs and the rotationless index}

We first explain for the reader more familiar with surface theory the ideal Whitehead graph and index list for a pseudo-Anosov mapping class $\psi$ on a closed surface $S$. Suppose that $v$ is a $k$-pronged foliation singularity and $\tilde{\psi}$ is a lift of $\psi$ to the universal cover $\widetilde{S}$ fixing a lift $\tilde{v}$ of $v$. Then $\widetilde{v}$ in fact lies inside of a principal region $P$ for the invariant lamination. The index list entry for $v$ would be $1-\frac{k}{2}$ and the ideal Whitehead graph component would be a $k$-gon. Equivalently, the ideal Whitehead graph component is the polygon formed by the lamination leaf lifts bounding the principal region $P$.

We remind the reader of the definition from [14] of the ideal Whitehead graph of a nongeometric fully irreducible outer automorphism. One can reference [19] and [14] for alternative definitions of the ideal Whitehead graph and its outer automorphism invariance.

Definition 2.21 (ideal Whitehead graph $\mathcal{I} \mathcal{W}(\varphi)$ ) Let $\varphi \in \operatorname{Out}\left(F_{r}\right)$ be a nongeometric fully irreducible outer automorphism with lifted attracting lamination $\tilde{\Lambda}$ (realized in $T_{+}$). To define the ideal Whitehead graph, start with the graph having a vertex for each distinct leaf endpoint and an edge connecting the vertices for the endpoints of each leaf. $\widetilde{\mathcal{I V}}(\varphi)$ is the union of the components with at least three vertices. $F_{r}$ acts freely, properly discontinuously, and cocompactly in such a way that the restriction to each component of $\widetilde{\mathcal{I} \mathcal{W}}(\varphi)$ has trivial stabilizer. The ideal Whitehead graph $\mathcal{I} \mathcal{W}(\varphi)$ is the quotient under this action, which one can note has only finitely many components. 
The ideal Whitehead graph has another interpretation in terms of singular leaves of the attracting lamination. For a fully irreducible $\varphi$, a leaf of the attracting lamination is singular if it shares a half-leaf with another leaf. Two half-leaves are asymptotic if they share a common ray. $\widetilde{\mathcal{I} W}(\varphi)$ has a vertex for each asymptotic class of half-leaves of singular leaves and an edge for each singular leaf of $\widetilde{\Lambda}$. The edges for two singular half-leaves share a vertex precisely when those half-leaves share an endpoint in the boundary. This definition also allows one to view $\widetilde{\mathcal{I W}}(\varphi)$ in any $F_{r}$-tree $T \in \operatorname{TT}\left(\varphi^{k}\right)$. Many details of the correspondence of these views will be explained in Remark 2.30.

Remark 2.22 As mentioned before, cut vertices of an ideal Whitehead graph yield representatives with PNPs. One can obtain such a representative from a stable representative by splitting open at cut vertices of the ideal Whitehead graph, as in [14, Lemma 4.3]. We use this in particular in Lemma 4.5.

The notion of an index ind $(\varphi)$ for a $\varphi \in \operatorname{Out}\left(F_{r}\right)$ was first introduced in [10]. This notion is not in general invariant under taking powers. The monograph [14] introduces the notion of a rotationless index (there just called the index sum) $i(\varphi)$ for a fully irreducible $\varphi \in \operatorname{Out}\left(F_{r}\right)$. It follows from [14, Lemma 3.4] that for rotationless nongeometric fully irreducible $\varphi \in \operatorname{Out}\left(F_{r}\right)$, the two notions differ only by a change of sign.

Definition 2.23 (index list and index sum) Let $\varphi \in \operatorname{Out}\left(F_{r}\right)$ be a nongeometric fully irreducible outer automorphism and $C_{1}, \ldots, C_{l}$ the connected components of $\mathcal{I} \mathcal{W}(\varphi)$. For each $j$, let $k_{j}$ denote the number of vertices of $C_{j}$. The index list for $\varphi$ is defined as

$$
\left(i_{1}, \ldots, i_{j}, \ldots, i_{l}\right)=\left(1-\frac{1}{2} k_{1}, \ldots, 1-\frac{1}{2} k_{j}, \ldots, 1-\frac{1}{2} k_{l}\right),
$$

where the list is rewritten to be in increasing order of absolute values with repetitions allowed. The rotationless index is then $i(\varphi)=\sum_{j=1}^{l} i_{j}$.

One can obtain the index list (hence rotationless index) from any PNP-free rotationless train track representative $g: \Gamma \rightarrow \Gamma$. The $k_{i}$ in (2) are replaced by the number of gates $k_{i}$ at the principal vertices $v_{i} \in \Gamma$. Since $\mathrm{g}$ is PNP-free, the principal vertices are precisely those periodic vertices with at least three gates. The index sum is therefore

$$
i(\varphi)=\sum_{\text {principal vertices } v}\left(1-\frac{1}{2} \#(\text { gates at } v)\right) .
$$

\subsection{Ageometrics}

The division of the set of nongeometric fully irreducibles into "ageometric" and "parageometric" outer automorphisms could be considered to have evolved out of a series 
of papers. In [11] Gaboriau and Levitt define the geometric index $\operatorname{ind}_{\text {geom }}(T)$ for an $\mathbb{R}$-tree equipped with a minimal, small $F_{r}$-action. They prove that the index satisfies the inequality $\frac{1}{2} \leq \operatorname{ind}_{\text {geom }}(T) \leq r-1$, with the equality $\operatorname{ind}_{\text {geom }}(T)=r-1$ realized precisely by "geometric trees". In [10] it is proved that, after replacing a fully irreducible $\varphi$ by a suitable positive power, one has $\operatorname{ind}_{\text {geom }}\left(T_{+}^{\varphi}\right)=2 \operatorname{ind}(\varphi)$. While it had been previously known that geometric fully irreducibles have geometric attracting tree, Levitt proved in [17] that even some nongeometric fully irreducibles have geometric attracting tree, hence creating a natural division of nongeometric fully irreducible outer automorphisms by their rotationless index. It will still be important for us that an ageometric fully irreducible $\varphi \in \operatorname{Out}\left(F_{r}\right)$ can be characterized by satisfying $0>i(\varphi)>1-r$. However, we also give an equivalent definition in terms of PNPs. The equivalence follows from the fact proved in [1, Theorem 3.2] that for a fully irreducible $\varphi \in \operatorname{Out}\left(F_{r}\right)$, the attracting tree $T_{+}^{\varphi}$ is geometric if and only if the "stable" train track representative of $\varphi$ contains a PNP. For interest's sake, we make one final remark that independently Handel and Mosher [13] and Guirardel [12] gave a further characterization that a fully irreducible outer automorphism is geometric if and only if both the attracting tree and repelling tree are geometric.

Definition 2.24 (ageometric) A fully irreducible outer automorphism is ageometric when a fully stable representative of a rotationless power has no Nielsen paths (closed or otherwise).

Remark 2.25 By [9, Lemma 3.28] every PNP of a rotationless train track representative is in fact an NP. Hence, a fully irreducible outer automorphism is ageometric if and only if one (hence every) fully stable representative of a (hence every) rotationless power has no NPs.

\subsection{Local decomposition of ideal Whitehead graphs}

The following definitions are from [14] and, as in that work, we make the following assumptions throughout:

- $\varphi \in \operatorname{Out}\left(F_{r}\right)$ is nongeometric fully irreducible,

- $T_{+}=T_{+}^{\varphi}$,

- $\Lambda=\Lambda_{\varphi}$,

- $T \in \widehat{\mathcal{A}}_{\varphi}$, and

- $f: T \rightarrow T_{+}$is a $\Lambda$-isometry. 
We further let $\Gamma:=T / F_{r}$. Unless specified, we do not assume that $T \in \mathrm{TT}(\varphi)$.

There is a partial ordering on the axis bundle which relies on the splitting of $\mathcal{I} \mathcal{W}(\varphi)$ into "stable Whitehead graphs".

We remind the reader of terminology and discussion found in [14, Section 3.3].

Definition 2.26 (principal points in trees) Given a branch point $b$ of $T_{+}$, the lifted ideal Whitehead graph $\widetilde{\mathcal{I} W}(\varphi)$ has one component, which we denote $\widetilde{\mathcal{I N}}_{b}(\varphi)$, whose edges, realized as lines in $T_{+}$, all contain $b$. This relationship gives a one-to-one correspondence between components of $\widetilde{\mathcal{I N}}(\varphi)$ and branch points of $T_{+}$. Given a branch point $b$ of $T_{+}$, we let $\widetilde{\mathcal{I N}}_{b}(\varphi ; T)$ denote the realization of $\widetilde{\mathcal{I N}}_{b}(\varphi)$ in $T$. This makes sense by viewing the ideal Whitehead graph in terms of the lamination leaves, as in Definition 2.21. We call a point $v$ in $T$ principal for $f$ if there exists a branch point $b$ of $T_{+}$such that $f(v)=b$ and $v$ is in $\widetilde{\mathcal{I W}}_{b}(\varphi ; T)$.

Remark 2.27 By definition, and since $f: T \rightarrow T_{+}$is a $\Lambda$-isometry, the restriction of $f$ to the principal points is surjective onto the set of principal points, ie branch points of $T_{+}$. Thus, $f$ injects principal vertices to branch points if and only if it bijects principal vertices to branch points.

Definition 2.28 (basepoints, singular rays, and principal directions) Let $l_{T}$ denote the realization in $T$ of a singular leaf $l$ of some component $\widetilde{\mathcal{I N}}_{b}(\varphi)$ of $\widetilde{\mathcal{I N}}(\varphi)$. Then there exists a unique principal point $v$ of $T$, called the basepoint of $l_{T}$, lying on $l_{T}$ and such that $f(v)=b$. Then $v$ divides $l$ into two rays. Rays obtained as such are called singular rays based at $v$. Given a principal point $v \in T$, the initial direction of a singular ray based at $v$ is called a principal direction at $v$.

Definition 2.29 (principal lifts and principal directions for principal lifts) In this definition, we add the additional assumption that $T$ is a train track, and we fix $g: \Gamma \rightarrow \Gamma$ to be a rotationless train track representative of $\varphi^{k}$ for some $k \geq 1$.

Given a principal vertex $\widetilde{v}$ in $T=\widetilde{\Gamma}$, there exists precisely one lift $\widetilde{g}$ of $g$ fixing $\widetilde{v}$. Such lifts are called principal lifts (see [9]).

There exists a one-to-one correspondence between the set of singular rays at $\tilde{v}$ and the set of fixed directions of $\tilde{g}$ at $\widetilde{v}$ (which are called the principal directions at $\tilde{v}$ for $\tilde{g}$ ). The correspondence can be seen as follows (further details can be found in [19]). Given a direction $d$ of an edge $E$ at a principal vertex $v$, the ray determined by $d$ is defined as $\widetilde{R}=\bigcup_{j=0}^{j=\infty} \widetilde{g}^{j}(E)$, where $\widetilde{g}$ is a lift, fixing $v$, of a rotationless train track representative $g: \Gamma \rightarrow \Gamma$ for some $\varphi^{k}$. 
Remark 2.30 As in Definition 2.29, $T \in \mathrm{TT}(\varphi)$ and $f: T \rightarrow T_{+}$is a $\Lambda$-isometry.

Recall from Definition 2.3 that a principal point downstairs either has at least three periodic directions or is the endpoint of a PNP. For a principal point downstairs, having at least three periodic directions, any lift is a principal point upstairs (with at least three singular rays determined by the singular directions, as in Definition 2.29). Additionally, since the directions are fixed, they give three distinct edges at $f(v)$, so that $f(v)$ is indeed a branch point of $T_{+}$.

Suppose that instead $v, w$ are the endpoints of an iNP $\rho$ for $g$. Then there exist at least two fixed directions at $v$ (one, which we call $E_{1}$, is a terminal edge of $\rho$ ) and at least two fixed directions at $w$ (one, which we call $E_{2}$, is the other terminal edge of $\rho$ ). For any lift $\tilde{\rho}$ of $\rho$ with terminal vertices $\widetilde{v}$ and $\widetilde{w}$, lifts of $v$ and $w$ respectively, $f$ sends $\tilde{v}$ and $\tilde{w}$ to a common point $b$ of $T_{+}$, which has at least three directions (one of which arises from the identification of $E_{1}$ and $E_{2}$ and the other two of which come from the distinct fixed directions at $v$ and $w$ ). Hence, $b$ is also a branch point of $T_{+}$. Notice that the rays constructed above from $E_{1}$ and $E_{2}$ are also asymptotic in $T$.

All principal points and principal directions in $T$ arise in one of these two ways.

Definition 2.31 (stable Whitehead graphs $\mathcal{S W}(\widetilde{v} ; T)$ and local Whitehead graphs $\mathcal{L W}(\tilde{v} ; T)$ for weak train tracks) Let $T$ be a weak train track. The local Whitehead graph $\mathcal{L W}(\tilde{v} ; T)$ will have a vertex for each direction at $\tilde{v}$ and an edge connecting the vertices corresponding to the pair of directions $\left\{d_{1}, d_{2}\right\}$ if the turn $\left\{d_{1}, d_{2}\right\}$ is taken by the realization in $T$ of a leaf of $\widetilde{\Lambda_{\varphi}}$. The stable Whitehead graph $\mathcal{S W}(\widetilde{v} ; T)$ at a principal point $\widetilde{v}$ will be the subgraph of $\mathcal{L W}(\widetilde{v} ; T)$ obtained by restricting to the principal directions. Equivalently, the stable Whitehead graph $\mathcal{S W}(\widetilde{v} ; T)$ at a principal point $v$ can be identified with the graph having one vertex for each singular ray $\widetilde{R}$ based at $v$ and an edge connecting the vertices corresponding to a pair of singular rays $\widetilde{R_{1}}, \widetilde{R_{2}}$ at $v$ if and only if $\widetilde{R_{1}} \cup \widetilde{R_{2}}$ is a singular leaf at $v$. One can reference [14] for further details.

Definition 2.32 (stable Whitehead graph $\mathcal{S W}(v ; \Gamma)$ and local Whitehead graph $\mathcal{L W}(v ; \Gamma)$ for train tracks) For a general weak train track $T$ and $\Gamma=T / F_{r}$, one can obtain $\mathcal{S} \mathcal{W}(v ; \Gamma)$ and $\mathcal{L W}(v ; \Gamma)$ from $\mathcal{S W}(\widetilde{v} ; T)$ and $\mathcal{L W}(\widetilde{v} ; T)$ by quotienting by the $F_{r}$-action. In the special case of a train track $T$ for $\varphi$, there is also a more direct construction of these Whitehead graphs in $\Gamma$, as follows.

The local Whitehead graph $\mathcal{L} \mathcal{W}(v ; \Gamma)$ at a point $v \in \Gamma$ has a vertex for each direction at $v$ and an edge connecting the vertices corresponding to the pair of directions $\left\{d_{1}, d_{2}\right\}$ if the turn $\left\{d_{1}, d_{2}\right\}$ is taken by the realization in $\Gamma$ of a leaf of $\Lambda_{\varphi}$. And 
the stable Whitehead graph $\mathcal{S W}(v ; \Gamma)$ at a principal point $v$ will be the subgraph of $\mathcal{L W}(v ; \Gamma)$ obtained by restricting to the periodic directions and the edges connecting them. Since each gate at a periodic vertex contains precisely one periodic direction, one can equivalently give this definition in terms of gates. To do this one retracts each gate to the periodic direction in that gate. Given two gates $g_{1}$ and $g_{2}$, the set of edges between directions in $g_{1}$ and $g_{2}$ is retracted to a single edge between $g_{1}$ and $g_{2}$.

Remark 2.30 explains the relationship between the different definitions of stable Whitehead graphs.

Given a train track map $g: \Gamma \rightarrow \Gamma$, the direction map $D g$ induces a simplicial map on both the local Whitehead graph and the stable Whitehead graph. We again denote these maps by $D g$.

Remark 2.33 Each stable Whitehead graph $\mathcal{S W}(\widetilde{v} ; T)$ sits inside of $\widetilde{\mathcal{I W}}(\varphi)$ : A vertex of $\mathcal{S W}(\widetilde{v} ; T)$ corresponds to a singular leaf $\widetilde{R}$ at $\widetilde{v}$ and the endpoint of this ray corresponds to a vertex of $\widetilde{\mathcal{I} W}(\varphi)$. An edge of $\mathcal{S W}(\widetilde{v} ; T)$ corresponds to a singular leaf based at $\widetilde{v}$. This leaf also gives an edge of $\widetilde{\mathcal{I} \mathcal{W}}(\varphi)$.

The following is a restatement of [14, Lemma 5.2], where information unnecessary for our purposes is left out.

Lemma 2.34 Suppose that $T$ is a weak train track and $f_{T}: T \rightarrow T_{+}$a $\Lambda$-isometry. Suppose that $b$ is a branch point of $T_{+}$and $\left\{\tilde{w}_{i}\right\} \subset T$ is the set of principal vertices mapped by $f_{T}$ to $b$. Then:

(1) $\widetilde{\mathcal{I N}}_{b}(\varphi ; T)=\bigcup \mathcal{S W}\left(\tilde{w}_{i} ; T\right)$.

(2) For each $i \neq j$, the intersection $\mathcal{S W}\left(\tilde{w}_{i} ; T\right) \cap \mathcal{S W}\left(\tilde{w}_{j} ; T\right)$ is at most one vertex. In the case where there is a vertex $P$ in the intersection, we have that $P$ is a cut point of $\widetilde{\mathcal{I W}}(\varphi)$, separating $\mathcal{S W}\left(\widetilde{w}_{i} ; T\right)$ from $\mathcal{S W}\left(\widetilde{w}_{j} ; T\right)$ in $\widetilde{\mathcal{I W}}(\varphi)$.

Definition 2.35 (local decompositions and splitting) In light of Remark 2.33 (and [14, Lemma 5.2]), the ideal Whitehead graph, realized in $T$, can be written as the union of the stable Whitehead graphs at the principal points. We call this its local decomposition.

Let $T, T^{\prime}$ be weak train tracks with $\Lambda$-isometries $f: T \rightarrow T_{+}$and $f^{\prime}: T^{\prime} \rightarrow T_{+}$. As in [14], one says $f$ splits as much as $f^{\prime}$ if the local decomposition $\widetilde{\mathcal{I W}}(\varphi)=$ $\bigcup \mathcal{S W}\left(v_{j} ; T\right)$ is at least as fine as the local decomposition $\widetilde{\mathcal{I} \mathcal{W}}(\varphi)=\bigcup \mathcal{S W}\left(w_{i} ; T^{\prime}\right)$. That is, for each principal vertex $v_{j}$ of $T$, there exists a principal vertex $w_{i}$ of $T^{\prime}$ such that $\mathcal{S W}\left(v_{j} ; T\right) \subset \mathcal{S W}\left(w_{i} ; T\right)$, where the inclusion takes place in $\widetilde{\mathcal{I} W}(\varphi)$, realized as a decomposition, as above. 
The following lemma is a consequence of the definitions in [14, Section 5.1].

Lemma 2.36 Let $\varphi \in \operatorname{Out}\left(F_{r}\right)$ be nongeometric fully irreducible and let $T, T^{\prime}$ be weak train tracks for $\varphi$ with $\Lambda$-isometries $f_{T}: T \rightarrow T_{+}$and $f_{T^{\prime}}: T^{\prime} \rightarrow T_{+}$. Then:

(A) If $f_{T}$ and $f_{T^{\prime}}$ are each injective on principal vertices, then they split equally.

(B) If $f_{T}$ is injective on principal vertices, then $f_{T^{\prime}}$ splits at least as much as $f_{T}$.

We will also use [14, Proposition 5.4], which we record here as Proposition 2.37.

Proposition 2.37 Let $\varphi \in \operatorname{Out}\left(F_{r}\right)$ be nongeometric fully irreducible. Then for any train track representative $g: \Gamma \rightarrow \Gamma$ for $\varphi$ with associated $\Lambda$-isometry $g_{\infty}: \widetilde{\Gamma} \rightarrow T_{+}$, there exists an $\varepsilon>0$ so that, if $f: T \rightarrow T_{+}$is any $\Lambda$-isometry, if $g_{\infty}$ splits at least as much as $f$, and if $\operatorname{Len}(T) \leq \varepsilon$, then there exists a unique equivariant edge-isometry $h: \widetilde{\Gamma} \rightarrow T$ such that $g_{\infty}=f \circ h$. Moreover, $h$ is a $\Lambda$-isometry.

\section{The stable axis bundle}

As mentioned above, the stable axis bundle was introduced in [14, Section 6.5] as an object of interest and is studied here as a means to a more general proof of our main theorem. For this purpose we establish here rigorously properties previously believed true.

Definition 3.1 (stable axis bundle) Let $\psi \in \operatorname{Out}\left(F_{r}\right)$ be ageometric fully irreducible. Then define

(4) $\operatorname{ST}(\psi):=\{\Gamma \in \mathrm{TT}(\psi) \mid \exists$ a fully stable train track representative

$$
g: \Gamma \rightarrow \Gamma \text { for } \psi\}
$$

The stable axis bundle is

$$
\mathcal{S} \mathcal{A}_{\varphi}=\overline{\bigcup_{k=1}^{\infty} \operatorname{ST}\left(\varphi^{k}\right)} .
$$

One can reformulate the stable axis bundle definition in terms of principal points:

Lemma 3.2 Let $g: \Gamma \rightarrow \Gamma$ be a rotationless train track representative of a positive power of an ageometric fully irreducible $\varphi \in \operatorname{Out}\left(F_{r}\right)$. Then the following are equivalent:

(1) $\varphi$ is fully stable.

(2) $\varphi$ has no Nielsen paths.

(3) The associated map $g_{\infty}: \widetilde{\Gamma} \rightarrow T_{+}$is injective on the set of principal points. 
Proof That (1) implies (2) is simply Definition 2.24. (2) implies (1) by the definition of a stable train track representative. We now show (2) implies (3). Assume $g$ is NP-free and that (3) does not hold, ie that there exist distinct points $\tilde{x}, \tilde{y} \in \widetilde{\Gamma}$ such that $g_{\infty}(\tilde{x})=g_{\infty}(\tilde{y})$. Then Proposition 2.19 tells us that we can take a path $\sigma$ from $\tilde{x}$ to $\tilde{y}$, project to $\Gamma$, and know that some $g_{\#}^{k}(\sigma)$ will either be trivial or an NP. However, it cannot be trivial since the endpoints are distinct and periodic. Hence, some power of $\sigma$ must be an NP, contradicting our assumption. We now prove that (3) implies (2). Suppose that $\Gamma$ has an NP $\sigma$. Let $\tilde{g}$ and $\tilde{\sigma}$ be lifts of $g$ and $\sigma$, respectively, so that $\tilde{g}$ preserves the endpoints $\tilde{x}, \tilde{y}$ of $\tilde{\sigma}$. Then Proposition 2.19 implies that $g_{\infty}(\tilde{x})=g_{\infty}(\tilde{y})$, ie that $g_{\infty}$ is not injective on the set of principal points.

Definition 3.3 (stable weak train track) Generalizing the lemma, one can define a weak train track $\Gamma$ to be stable if there exists a $\Lambda$-isometry $\widetilde{\Gamma} \rightarrow T_{+}$which is injective on the set of principal points. We denote the set of stable weak train tracks for a given fully irreducible $\varphi$ by $\operatorname{SWTT}(\varphi)$.

Proposition 3.11 will then imply that $\mathcal{S A}_{\varphi}$ and $\operatorname{SWTT}(\varphi)$ are in fact the same set.

Definition 3.4 (weak periodic Nielsen path) A weak periodic Nielsen path in a weak train track $T$ is a homotopically nontrivial path in $T$ whose endpoints are principal points with the same image in $T_{+}$.

One can then also characterize stable weak train tracks by their lack of weak PNPs:

Lemma 3.5 A weak train track $T$ is stable if and only if it has no weak PNPs.

Proof If $T$ is a stable weak train track, then it is injective on principal points. Hence, the endpoints of a weak PNP in $T$ would be the same, but this is impossible since $T$ is a tree.

Suppose that $T$ is a weak train track with distinct principal points $v_{1}, v_{2}$ in $T$ having the same image in $T_{+}$. Since $T$, being a tree, is connected, there exists some path from $v_{1}$ to $v_{2}$ in $T$. This path would be a weak PNP. Hence, if $T$ is a weak train track, it is injective on principal points.

Lemma 3.6 Suppose $g: \Gamma \rightarrow \Gamma$ is a fully stable train track representative of a rotationless power $\varphi^{R}$ of a fully irreducible $\varphi \in \operatorname{Out}\left(F_{r}\right)$. If the rotationless index satisfies $i(\varphi)=\frac{3}{2}-r$, then $g$ has a unique illegal turn. 
Proof As laid out in Definition 2.24 and the preceding paragraph, from results of [1] it follows that $\varphi$ is ageometric if and only if $i(\varphi) \geq \frac{3}{2}-r$ if and only if each stable train track representative of $\varphi$ is PNP-free.

For simplicity, in what follows, we define

$$
\mathrm{GI}(g)=\sum_{\text {vertices } v}\left(1-\frac{1}{2} \#(\text { gates of } v)\right) .
$$

Recall also (from Definition 2.23) that the rotationless index $i(\varphi)$ is the same sum as $\mathrm{GI}(g)$ but with the terms indexed by nonperiodic vertices with $\geq 3$ gates removed. Hence, in particular, $\mathrm{GI}(g) \leq i(\varphi)$. We also have

$$
\begin{aligned}
1-r=\chi(\Gamma) & =\# \text { Vertices }-\# \text { Edges } \\
& =\# \text { Vertices }-\frac{1}{2} \sum_{\text {gates } D} \operatorname{card}(D) \\
& =\mathrm{GI}(g)+\frac{1}{2} \sum_{\text {gates } D}(1-\operatorname{card}(D)) .
\end{aligned}
$$

Thus,

$$
(1-r)-\mathrm{GI}(g)=\frac{1}{2} \sum_{\text {gates } D}(1-\operatorname{card}(D)) .
$$

Each term on the right-hand side of (6) is nonpositive and is zero if and only if the gate $D$ consists of a single direction. Thus, $\mathrm{GI}(g)=1-r$ if and only if each gate has cardinality 1 . This is true if and only if there are no illegal turns, which is impossible as $\varphi$ is fully irreducible, hence of infinite order. Thus, $\operatorname{GI}(g)>1-r$ and, since $\operatorname{GI}(g)$ can only take half-integer values, $\mathrm{GI}(g) \geq \frac{3}{2}-r$. Hence, under the assumption that $i(\varphi)=\frac{3}{2}-r$, we have

$$
\frac{3}{2}-r \leq \mathrm{GI}(g) \leq i(\varphi)=\frac{3}{2}-r .
$$

Now, GI $(g)=\frac{3}{2}-r$ precisely when each gate has cardinality 1 , except a single gate of cardinality 2. Equivalently, $\mathrm{GI}(g)=\frac{3}{2}-r$ if and only if $g$ has a unique illegal turn.

Remark 3.7 The proof of the above lemma implies that, given a fully stable train track representative $g: \Gamma \rightarrow \Gamma$ of a rotationless power $\varphi^{R}$ of a fully irreducible $\varphi \in \operatorname{Out}\left(F_{r}\right)$ satisfying $i(\varphi)=\frac{3}{2}-r$, we have

$$
i(\varphi)=\mathrm{GI}(g) .
$$

The following corollary, while not used in what follows, may be of independent interest. 
Corollary 3.8 Let $\varphi \in \operatorname{Out}\left(F_{r}\right)$ be a fully irreducible outer automorphism with rotationless index satisfying $i(\varphi)=\frac{3}{2}-r$. Then there exists a rotationless power $\varphi^{R}$ of $\varphi$ with a fully stable train track representative $g: \Gamma \rightarrow \Gamma$ such that each vertex of $\Gamma$ is principal with respect to $g$ and all but one direction of $\Gamma$ is fixed by $D g$.

Proof Let $g^{\prime}: \Gamma^{\prime} \rightarrow \Gamma^{\prime}$ be a stable train track representative of a rotationless power $\varphi^{R}$. Such a representative exists by Lemmas 3.7 and 3.8 of [4] and the intervening paragraph. Amongst all such representatives we may assume that $\Gamma^{\prime}$ has the minimal number of vertices.

By Definition 2.6, $g^{\prime}$ is fully stable. Passing to a higher power, we may assume that $g^{\prime}$ fixes every periodic vertex in $\Gamma^{\prime}$, that $D g^{\prime}$ fixes every periodic direction at each periodic vertex, and that $g^{\prime}$ fixes the image of every nonperiodic vertex, and hence every nonperiodic vertex has no vertex in its preimage. If any valence- 2 vertex is nonperiodic then that vertex may be removed from the list of vertices, contradicting minimality. It follows that every vertex of valence 2 is fixed.

By the proof of Lemma 3.6 (see Remark 3.7) we have $i\left(\varphi^{R}\right)=\mathrm{GI}\left(g^{\prime}\right)$. In particular no nonperiodic vertex can have more than two gates. Since $g^{\prime}$ has only one illegal turn (by Lemma 3.6), there exists at most one vertex of valence $\geq 3$ with only two gates. Hence, there exists at most one nonperiodic vertex of valence $\geq 3$.

Let $v \in \Gamma$ be the vertex at which $g^{\prime}$ has an illegal turn $\left\{d_{1}, d_{2}\right\}$. Let $g^{\prime \prime}: \Gamma^{\prime \prime} \rightarrow \Gamma^{\prime \prime}$ be the train track representative of $\varphi^{R}$ obtained by folding the illegal turn of $g^{\prime}$ in the sense of [4, page 11], so there is a factorization

$$
g^{\prime}: \Gamma^{\prime} \stackrel{g_{1}}{\longrightarrow} \Gamma^{\prime \prime} \stackrel{g_{2}}{\longrightarrow} \Gamma^{\prime}
$$

where $g_{1}$ folds the illegal turn, and $g^{\prime \prime}=g_{1} \circ g_{2}: \Gamma^{\prime \prime} \rightarrow \Gamma^{\prime \prime}$. The fold $g_{1}$ identifies initial segments $\eta_{1}, \eta_{2}$ of the oriented edges $E_{1}, E_{2}$ representing $\left\{d_{1}, d_{2}\right\}$; these are the maximal initial segments that are identified by $g^{\prime}$, and $g_{1}$ must be a full fold, meaning that $\eta_{i}=E_{i}$ for some $i=1,2$, for otherwise $\eta_{1}, \eta_{2}$ are both proper and their terminal endpoints are identified to a new valence- 3 vertex of $\Gamma^{\prime \prime}$ having three gates, contradicting that $i\left(\varphi^{R}\right)=\mathrm{GI}\left(g^{\prime}\right)=\mathrm{GI}\left(g^{\prime \prime}\right)$. Since $g_{1}$ is a full fold it follows that $\Gamma^{\prime \prime}$ has no more vertices than $\Gamma^{\prime}$. Also, $g^{\prime \prime}$ is fully stable, because folding in the sense of [4, page 11] cannot create periodic Nielsen paths. It follows by minimality that $\Gamma^{\prime \prime}$ has the same number of vertices as $\Gamma^{\prime}$, and so $g_{1}$ is a proper full fold, identifying all of $E_{1}$ or $E_{2}$ with a proper initial segment of the other.

The proof now breaks into the following two cases:

(1) There are no nonperiodic vertices.

(2) There is a single nonperiodic vertex, and it has two gates and the unique illegal turn, and so it must be equal to $v$. 
Case 1 In this case there is no vertex of valence 2, because any such vertex has no vertex in its preimage and hence may be removed, contradicting minimality. If $v$ has valence $\geq 4$ then we are done, because each vertex is a fixed vertex with at least three fixed directions and so is principal, in addition to the illegal turn containing the only nonfixed direction. If $v$ has valence 3 then, after the proper full fold $g_{1}: \Gamma^{\prime} \rightarrow \Gamma^{\prime \prime}$, the vertex $w=g_{1}(v)$ has valence 2 in $\Gamma^{\prime \prime}$ and has no vertex in its $g^{\prime \prime}$ preimage, thus $w$ may be removed from the list of vertices of $\Gamma^{\prime \prime}$, contradicting minimality.

Case 2 The vertex $v$ has valence 3, and so as in Case 1 the vertex $w=g_{1}(v)$ has valence 2 in $\Gamma^{\prime \prime}$ and has no vertex in its $g^{\prime \prime}$ preimage, leading again to a contradiction with minimality.

Lemma 3.9 Suppose $\varphi \in \operatorname{Out}\left(F_{r}\right)$ is ageometric fully irreducible with $i(\varphi)=\frac{3}{2}-r$. We view points in outer space as free, simplicial $F_{r}$-trees. Then, for each $T \in$ $\bigcup \operatorname{ST}\left(\varphi^{k}\right)$, the $\Lambda$-isometry $\mathcal{I}: T \rightarrow T_{+}$is unique.

Proof Let $\varphi \in \operatorname{Out}\left(F_{r}\right)$ be as in the lemma and have attracting lamination $\Lambda$, ie $\Lambda=\Lambda_{\varphi}$. Consider a point in outer space viewed as a free, simplicial $F_{r}$-tree $T$. In [14], Handel and Mosher define an orientation of $\tilde{\Lambda}$ in $T$ as an $F_{r}$-equivariant choice of orientation on each leaf of $\tilde{\Lambda}$ satisfying that the orientations of leaves $L_{T}$ and $L_{T}^{\prime}$ agree on their intersection $L_{T} \cap L_{T}^{\prime}$, provided the intersection contains a nontrivial interval. By birecurrence, an orientation on $\widetilde{\Lambda}$ is determined by an orientation of any of its leaves. Also note that an orientation of $\tilde{\Lambda}$ in $T$ induces a well-defined $F_{r}$-equivariant orientation on each edge of $T$, hence on its quotient graph $\Gamma=T / F_{r}$. In [14, Theorem 5.8] the authors prove that the $\Lambda$-isometry $\mathcal{I}: T \mapsto T_{+}$is unique if $\tilde{\Lambda}$ is nonorientable. Hence, we can assume $\tilde{\Lambda}$ is orientable and fix an orientation.

By a positive gate we will mean a gate that the attracting lamination only exits (and never enters) the vertex through. A negative gate will mean a gate that the attracting lamination only enters (and never exits) the vertex through. Each gate is either positive or negative. A direction in a positive gate will be called positive and a direction in a negative gate will be called negative. Notice that, for an orientable lamination realized in $\Gamma$, for each edge of $\Gamma$, the direction of the edge at one vertex is positive and the direction of the edge at the other vertex is negative.

Now let $T$ specifically represent a point in $\bigcup \operatorname{ST}\left(\varphi^{k}\right)$ and again let $\Gamma=T / F_{r}$. Thus, there exists a fully stable train track representative $g: \Gamma \rightarrow \Gamma$ of some rotationless power $\varphi^{R}$. According to [14, Theorem 5.8c(iii)], $\mathcal{I}: T \rightarrow T_{+}$is unique if and only if there exist vertices $v, w \in T$ such that $v$ has $\geq 2$ positive gates with respect to $\mathcal{I}$ and $w$ has $\geq 2$ negative gates with respect to $\mathcal{I}$. Thus, by symmetry, it suffices to prove it impossible for each vertex to have a unique positive gate. 
For the sake of contradiction we assume each vertex has only one positive gate. We will use the fact that, in the absence of PNPs, the rotationless index is computed from the gates (see Remark 3.7). In particular, we will also use the fact that, since $i(\varphi)=\frac{3}{2}-r$, there is only one illegal turn (see Lemma 3.6), ie there is a unique gate with two directions and all other gates have only one. Each vertex with $\geq 3$ gates has $\geq 2$ negative gates. Also, the total number of positive vertex directions in $\Gamma$ and of negative vertex directions in $\Gamma$ must be equal in order for them to correspond to a set of edge orientations.

We restrict our attention to vertices of valence $\geq 3$. We consider separately the cases where $\Gamma$ has only one such vertex and where $\Gamma$ has more than one such vertex. If $\Gamma$ has only one such vertex, then it would have to have $2 r-2$ negative gates. Thus, it would have $\geq 2 r-2$ negative directions and $\leq 2$ positive directions. For $r \geq 3$ this makes having an equal number of positive and negative directions impossible. So suppose $\Gamma$ has $k \geq 2$ such vertices. Then $\Gamma$ would have $\geq 2 k$ negative gates by the previous paragraph. Thus, it would have $\geq 2 k$ negative directions and at most $k+1$ positive directions. This is a contradiction, as above, unless $k=1$.

Before proceeding with Proposition 3.11, we recall from [14, Proposition 5.5] the following. Most of the notation is described in Definition 2.31 and Definition 2.26; however, we need one more definition. We gave in Definition 2.2 the definition of a periodic point in a train track. One can define the set of periodic points in $T_{+}$using any $\Lambda$-isometry $f: T \rightarrow T_{+}$on a train track $T$. The periodic points in $T_{+}$will be the images under $f$ of the periodic points in $T$. This definition is equivalent to the intrinsic definition, involving automorphisms representing $\varphi$, given in [14, Section 5.3].

Proposition 3.10 [14] Let $T$ be a weak train track and $f: T \rightarrow T_{+}$a $\Lambda$-isometry. Then the following are necessary and sufficient conditions for the existence of a train track representative $g: \Gamma \rightarrow \Gamma$ of $\varphi^{k}$, for some $k$, such that $\Gamma=T / F_{r}$ and $f=g_{\infty}$ (ie for $\Gamma$ to be a train track for $\varphi^{k}$ ):

(1) For every vertex $w$ of $T, f(w) \in T_{+}$is a periodic point.

(2) For every vertex $y$ of $T$, if $f(y)=b$ is a branch point of $T_{+}$then there exists a principal vertex $w$ of $T$ such that

$$
D f(\mathcal{L W}(y ; T)) \subset D f(\mathcal{S W}(w ; T)) \subset \widetilde{\mathcal{I N}}_{b}(\varphi) .
$$

Proposition 3.11 Suppose that $\varphi \in \operatorname{Out}\left(F_{r}\right)$ is an ageometric, fully irreducible outer automorphism with $i(\varphi)=\frac{3}{2}-r$. Then:

(A) $\mathcal{S A}_{\varphi}$ is the set of stable weak train tracks for $\varphi$.

(B) $\mathcal{S A}_{\varphi}$ is a nonempty, closed, $\varphi$-invariant subset of $\mathcal{A}_{\varphi}$. 
Proof For the purposes of this proof, given any weak train track $T^{\prime}$ with associated $\Lambda$-isometry $T^{\prime} \rightarrow T_{+}$, we call a point in $T^{\prime}$ mapping to a periodic point in $T_{+}$ preperiodic.

We first show, via applying Proposition 3.10, that $\operatorname{SWTT}(\varphi)$ is contained in $\overline{\operatorname{UST}\left(\varphi^{k}\right)}$. We cannot directly apply Proposition 3.10 to a given $T \in \operatorname{SWTT}(\varphi)$ (with $\Lambda$-isometry $f_{T}: T \rightarrow T_{+}$) because $T$ may fail to satisfy the first of the necessary and sufficient conditions for each vertex being $T_{+}$preperiodic. Hence, we approximate $T$ by performing the operation in the proof of [14, Corollary 5.6] of eliminating one at a time those vertices $w$ which are not $T_{+}$preperiodic, via small folds of directions at $w$ having the same image. In fact, the folds can be chosen sufficiently small to avoid interaction with any principal vertices. In particular, for the $T^{\prime}$ obtained, the injectivity of $f_{T^{\prime}}$ on the set of principal vertices is unaffected, and the weak train tracks $f_{T^{\prime}}: T^{\prime} \rightarrow T_{+}$ obtained will still be stable. Thus, we can apply Proposition 3.10 to approximate $T$ by stable weak train tracks provided that the $T^{\prime}$ satisfy Proposition 3.10(2), and hence are train tracks. That is, for each vertex $y$ of $T^{\prime}$ such that $f(y)=b$ for some branch point of $T_{+}$, we need that there exists a principal vertex $w$ of $T^{\prime}$ such that

$$
D f_{T^{\prime}}\left(W\left(y ; T^{\prime}\right)\right) \subset D f_{T^{\prime}}\left(\mathcal{S W}\left(w ; T^{\prime}\right)\right) \subset \widetilde{\mathcal{I W}}_{b}(\varphi) .
$$

First notice that, because $f_{T^{\prime}}: T^{\prime} \rightarrow T_{+}$is a stable weak train track, for each principal point $w \in T^{\prime}$ and $b=f_{T^{\prime}}(w)$ we have that $D f_{T^{\prime}}\left(\mathcal{S W}\left(w ; T^{\prime}\right)\right)=\widetilde{\mathcal{I N}}_{b}(\varphi)$. This follows from the facts that

$$
D f_{T^{\prime}}\left(\mathcal{S W}\left(w ; T^{\prime}\right)\right) \subset \widetilde{\mathcal{I N}}_{b}(\varphi) \subset \bigcup_{\text {principal } x} D f_{T^{\prime}}\left(\mathcal{S W}\left(x ; T^{\prime}\right)\right)
$$

and that, since $f_{T^{\prime}}$ is injective on principal vertices, no leaf of

$$
\bigcup_{\text {principal } x} D f_{T^{\prime}}\left(\mathcal{S W}\left(x ; T^{\prime}\right)\right)-D f_{T^{\prime}}\left(\mathcal{S W}\left(w ; T^{\prime}\right)\right)
$$

can be contained in $\widetilde{\mathcal{I W}}_{b}(\varphi)$. We know that $D f_{T^{\prime}}\left(W\left(y ; T^{\prime}\right)\right) \subset \widetilde{\mathcal{I W}}_{b}(\varphi)$ since it contains all leaves passing through $b$. Hence, (8) holds and Proposition 3.10(2) is satisfied. So the $T^{\prime}$ are both stable weak train tracks and train tracks and hence are stable train tracks by Lemma 3.2. Since they approximate $T$, we have that $T \in \overline{\mathrm{UST}\left(\varphi^{k}\right)}$. Hence, $\operatorname{SWTT}(\varphi) \subset \overline{\bigcup \operatorname{ST}\left(\varphi^{k}\right)}$, as desired.

Since each stable train track is in $\operatorname{SWTT}(\varphi)$, we are left to show for (A) that $\operatorname{SWTT}(\varphi)$ is closed. In other words, we need that each $T \in \overline{\operatorname{SWTT}(\varphi)}$ is in fact in $\operatorname{SWTT}(\varphi)$, ie that the associated $\Lambda$-isometry $T \rightarrow T_{+}$is injective on principal points. Notice that it also suffices to show this in $\widehat{\mathrm{CV}}_{r}$, as the projection to $\mathrm{CV}_{r}$ will then also be closed. Sometimes in what follows we will use the same notation for a tree and its projection. 
Let $T$ be in the closure and $T_{i}$ a sequence of stable weak train tracks converging to $T$. Take a subsequence, if necessary, so that all $T_{i}$ are in the same open cell. Notice that, if $T$ is not in the open cell containing the $T_{i}$, then it is in a face of the cell. Let $f_{i}^{+}$ denote the $\Lambda$-isometry $T_{i} \rightarrow T_{+}$.

Let $X=\bigcup\left\{T_{i}\right\} \cup T$. Then $X$ is a compact subset of $\widehat{\mathcal{A}_{\varphi}}$. Hence, since the length function is continuous, there is an upper bound on the length of a $F_{r}$-tree in $X$. By Proposition 2.17, for each $F_{r}$-tree $R$ in $\widehat{\mathcal{A}}_{\varphi}$ and each integer $k$, we have $\operatorname{Len}\left(\varphi^{k}(R)\right)=$ $\lambda(\varphi)^{-k} \operatorname{Len}(R)$. Thus, given any $\varepsilon$, there exists a $k_{\varepsilon}$ such that, for all $i$, we have $\operatorname{Len}\left(\varphi^{k_{\varepsilon}}\left(T_{i}\right)\right)<\varepsilon$. Because applying $\varphi^{k_{\varepsilon}}$ does not change full stability (just acts as a change of marking) it is safe in what follows to replace $\varphi^{k_{\varepsilon}}\left(T_{i}\right)$ with $T_{i}$, to replace $\varphi^{k_{\varepsilon}}(T)$ with $T$, etc.

By the arguments of the previous paragraph, we can assume that $\operatorname{Len}\left(T_{i}\right), \operatorname{Len}(T)<\varepsilon$ and apply Proposition 2.37 as follows. Since the $\Lambda$-isometry for each $F_{r}$-tree in $\left\{T_{i}\right\}$ is injective on principal points, we can choose some $F_{r}$-tree $S$ in $\mathcal{A}_{\varphi} \cap\left(\bigcup \operatorname{ST}\left(\varphi^{k}\right)\right)$, with $\Lambda$-isometry $s: S \rightarrow T_{+}$(also injective on principal points), such that $S$ satisfies the following condition: for each $T_{i}$, there exists a $\Lambda$-isometry $l_{i}: S \rightarrow T_{i}$ such that each arrow in the following diagram represents a $\Lambda$-isometry:

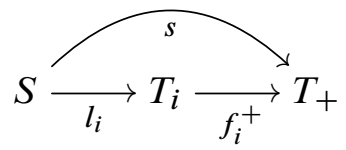

Since $T$ is in the closure of the cell containing the $T_{i}$, one can obtain $T$ from each $T_{i}$ via a quotient map $q_{i}: T_{i} \rightarrow T$, affine on edges. Let $m_{i}=q_{i} \circ l_{i}$ :

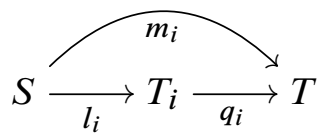

For each $i$, let $G_{i}:=T_{i} / F_{r}$ denote the quotient graph of $T_{i}$ and let $q_{i}^{\prime}: G_{i} \rightarrow G$ be the induced quotient map. Since all $T_{i}$ are in the same open cell, there exists a family of marked homeomorphisms $g_{j i}: G_{i} \rightarrow G_{j}$, affine on each edge, so that $g_{k j} \circ g_{j i}=g_{k i}$. For each $G_{i}$, choose an indexing $\left\{e_{i}^{\alpha}\right\}$ of the edge set of $G_{i}$ so that $g_{j i}\left(e_{i}^{\alpha}\right)=e_{j}^{\alpha}$. In a well-defined manner we can give each edge $q_{i}^{\prime}\left(e_{i}^{\alpha}\right)$ in $G$ the label $e^{\alpha}$. (Note that, for some $e_{i}^{\alpha}$, we have that $q_{i}^{\prime}\left(e_{i}^{\alpha}\right)$ is just a point, but we are only concerned with cases where it is an edge.)

Since each $l_{i}$ is a $\Lambda$-isometry, and the lengths of the $\left\{e_{i}^{\alpha}\right\}$ converge to the lengths of the $e^{\alpha}$, the $m_{i}$ converge to a $\Lambda$-isometry $m: S \rightarrow T$. Then $g \circ m: S \rightarrow T_{+}$is a 
composition of $\Lambda$ isometries, hence is a $\Lambda$-isometry. By Lemma 3.9, $s=g \circ m$ :

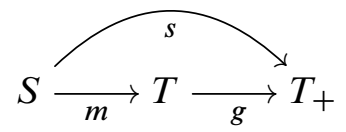

Recall that, by definition, principal points of an $F_{r}$-tree $R$ in $\mathcal{A}_{\varphi}$ map via the $\Lambda$ isometry $R \rightarrow T_{+}$to branch points in $T_{+}$. What we need to show is that the set of principal points of $T$ is mapped injectively via $g$ into the set of branch points of $T_{+}$, and $T \in \operatorname{SWTT}(\varphi)$, as desired. So, in particular, we need to show that, given principal points $v, w \in T$ with $g(v)=g(w)$, we have $v=w$.

Let $v, w \in T$ be principal points such that $g(v)=g(w)=b$ and let $l$ and $l^{\prime}$ be leaves of $\widetilde{\mathcal{I W}}_{b}(\varphi)$ such that $v$ is the basepoint of the realization $l_{T}$ of $l$ in $T$ and $w$ is the basepoint of the realization $l_{T}^{\prime}$ of $l^{\prime}$ in $T$. Let $b_{S}$ be the basepoint of the realization $l_{S}$ of $l$ in $S$ and let $b_{S}^{\prime}$ be the basepoint of the realization $l_{S}^{\prime}$ of $l^{\prime}$ in $S$. Then $b_{S}=b_{S}^{\prime}$, as $s$ is injective on principal points and $s\left(b_{S}\right)=b=s\left(b_{S}^{\prime}\right)$. Now, since $m$ is a $\Lambda$-isometry, $m$ maps $l_{S}$ isometrically onto $l$ and $l_{S}^{\prime}$ isometrically onto $l^{\prime}$. Also, $m\left(b_{S}\right)=v$ since $m$ maps $l_{S}$ isometrically onto $l_{T}$ and $v$ is the only point on $l_{T}$ mapped to $b$. Similarly, $m\left(b_{S}^{\prime}\right)=w$. Thus $v=w$, as desired, and the proof that $\operatorname{SWTT}(\varphi)$, and hence $\mathcal{S A}_{\varphi}$, is closed is complete.

$\operatorname{SWTT}(\varphi)$ is nonempty since $\varphi$ has a rotationless power with a stable representative (obtained, for example, via the stabilization algorithm of [4]). Since $\mathcal{S} \mathcal{A}_{\varphi}$ is a closure, hence closed, we are left to show that $\operatorname{SWTT}(\varphi)$ is $\varphi$-invariant. That $\bigcup \operatorname{ST}\left(\varphi^{k}\right)$ is invariant follows from the fact that changing the marking does not change stability. $\mathcal{S A}_{\varphi}$ is then invariant since the action is continuous.

\section{The proof}

According to Theorem 1.2 of [14], $\mathcal{A}_{\varphi}$ is proper homotopy equivalent to a line. In fact, there are distinguished lines from which it is possible to get to any point in $\mathcal{A}_{\varphi}$ by a combination of folding and of collapsing PNPs. These distinguished lines are periodic fold lines for representatives with the maximum number of PNPs possible. The strategy of our proof is to show when $\mathcal{A}_{\varphi}$ consists of only a single such fold line.

We start with a sequence of two lemmas immediately revealing the necessity for the rotationless index to be $\frac{3}{2}-r$.

Lemma 4.1 Suppose $g: \Gamma \rightarrow \Gamma$ is a fully stable train track representative of a rotationless power $\varphi^{R}$ of a fully irreducible $\varphi \in \operatorname{Out}\left(F_{r}\right)$. If the rotationless index satisfies 
$i(\varphi)>\frac{3}{2}-r$, then by following from $\Gamma$ a Stallings fold decomposition of $g$, one reaches a point $\Gamma^{\prime} \in \mathrm{CV}_{r}$ such that there exists a fully stable train track representative $g^{\prime}: \Gamma^{\prime} \rightarrow \Gamma^{\prime}$ of some power $\varphi^{R}$ with more than one illegal turn.

Proof As explained in the opening passage of Section 2.9, $\varphi$ is ageometric if and only if $i(\varphi) \geq \frac{3}{2}-r$. We thus can assume each stable representative is PNP-free.

We suppose $i(\varphi)>\frac{3}{2}-r$ and prove the lemma. The first observation we use is that $\mathrm{GI}(g) \leq i(\varphi)$, with equality if and only if each vertex with $\geq 3$ gates is fixed. The second observation is that the following three statements are equivalent:

(ii) Either there exist gates $D_{1}, D_{2}$ with $\operatorname{card}\left(D_{1}\right), \operatorname{card}\left(D_{2}\right)>1$ or there exists some gate $D$ with $\operatorname{card}(D)>2$.

(iii) $g$ has $\geq 2$ illegal turns.

Let $g: \Gamma \rightarrow \Gamma$ be any rotationless train track representative of a $\varphi^{R}$. If $g$ has $\geq 2$ illegal turns, then (iii) is proved. So we suppose $g$ has only one illegal turn. As above, $\mathrm{GI}(g)=\frac{3}{2}-r$. So $i(\varphi)>\mathrm{GI}(g)$ and $\Gamma$ must have a nonperiodic vertex with $\geq 3$ gates. Any such vertex $v$ is preperiodic.

By iterating a Stallings fold decomposition of $g: \Gamma \rightarrow \Gamma$ we obtain a periodic fold line as in Section 2.7.2 (see (1), in particular).

Let $k$ be such that $g_{k+1} \circ \cdots \circ g_{1}(v)=g_{k+1} \circ \cdots \circ g_{1}(w)$, while $g_{k} \circ \cdots \circ g_{1}(v) \neq$ $g_{k} \circ \cdots \circ g_{1}(w)$. For each $i$ let $v_{i}$ denote $g_{i} \circ \cdots \circ g_{1}(v)$, let $w_{i}$ denote $g_{i} \circ \cdots \circ g_{1}(w)$, and let

$$
f_{i}=g_{i} \circ \cdots \circ g_{1} \circ g_{K} \circ \cdots \circ g_{i+1}: \Gamma_{i} \rightarrow \Gamma_{i} .
$$

Notice that the fold map $g_{k}: \Gamma_{k} \rightarrow \Gamma_{k+1}$ conjugates $f_{k}$ to $f_{k+1}$, ie $g_{k+1} f_{k}=$ $f_{k+1} g_{k+1}$. Also, the $g_{k}$ bijectively map the periodic directions of the $w_{i}$ because periodic directions cannot be identified (as they are in distinct gates) and $g$ bijectively maps the periodic directions.

In $\Gamma_{k}$, the vertex $v_{k}$ is preperiodic (and not periodic) and has $\geq 3$ gates, while the vertex $w_{k}$ is fixed with $\geq 3$ gates. To identify $v_{k}$ and $w_{k}$, the fold $g_{k+1}: \Gamma_{k} \rightarrow \Gamma_{k+1}$ must be an improper full fold, which fully folds two oriented edges $E, E^{\prime}$ having the same initial vertex and having terminal vertices $v_{k}, w_{k}$ respectively. We have $g_{k+1}(E)=g_{k+1}\left(E^{\prime}\right)=E^{\prime \prime}$ with terminal vertex $g_{k+1}\left(v_{k}\right)=g_{k+1}\left(w_{k}\right)=w_{k+1}$. At $v_{k}$ there are $\geq 2$ directions, namely $d$ and $d^{\prime}$, not in the same gate as each other nor in the same gate as the terminal direction of $E$. It follows that $g_{k+1}(d)$ and $g_{k+1}\left(d^{\prime}\right)$ are two directions at $w_{k+1}$ not in the same gate as each other, and they are not periodic 
directions. Therefore, the two gates at $w_{k+1}$ containing $g_{k+1}(d)$ and $g_{k+1}\left(d^{\prime}\right)$ must each contain $\geq 1$ other direction, namely some periodic direction. Therefore, the train track representative $f_{k+1}$ has $\geq 2$ gates each of cardinality $\geq 2$. This proves that $g^{\prime}=f_{k+1}: \Gamma_{k+1} \rightarrow \Gamma_{k+1}$ is the desired fully stable train track representative with more than one illegal turn.

Comment 4.2 Notice that Lemma 4.1 does not assert that $\Gamma$ itself has more than one illegal turn. This motivates a question which may shed light on whether local dimension is constant on the axis bundle: does there exist a fully irreducible $\varphi \in \operatorname{Out}\left(F_{r}\right)$ and two fully stable train track representatives $g_{1}: \Gamma_{1} \rightarrow \Gamma_{1}$ and $g_{2}: \Gamma_{2} \rightarrow \Gamma_{2}$ such that $\Gamma_{1}$ has only one illegal turn and $\Gamma_{2}$ has more than one?

Lemma 4.3 Suppose that $\varphi \in \operatorname{Out}\left(F_{r}\right)$ is ageometric and fully irreducible. If $i(\varphi)>$ $\frac{3}{2}-r$, then, for each $k \geq 1$, each point in $\bigcup \mathrm{ST}\left(\varphi^{k}\right)$ is contained in at least two distinct periodic fold lines.

Proof Suppose $\varphi \in \operatorname{Out}\left(F_{r}\right)$ is ageometric and fully irreducible with rotationless index $i(\varphi)>\frac{3}{2}-r$. Then each $\varphi^{k}$ is also ageometric and fully irreducible with the same rotationless index. Let $\Gamma$ be a point of $\bigcup \operatorname{ST}\left(\varphi^{k}\right)$ and $f: \Gamma \rightarrow \Gamma$ a fully stable rotationless train track representative of some $\varphi^{k}$. By Lemma 4.1, we can fold from $\Gamma$ to an $X$ on which there exists a fully stable representative $g$ of $\varphi^{k}$ with more than one illegal turn, and that is in fact conjugate to $f$ by precisely the folds taken to move from $\Gamma$ to $X$.

For any illegal turn $\left\{d, d^{\prime}\right\}$ in $X$, consider its forward orbit $\left\{D g^{i}(d), D g^{i}\left(d^{\prime}\right)\right\}_{i \geq 0}$. There is a minimal value of $i \geq 1$ for which the turn $\left\{D g^{i}(d), D g^{i}\left(d^{\prime}\right)\right\}$ is degenerate. It follows that there exists an illegal turn $\left\{d, d^{\prime}\right\}$ in $X$ whose immediate forward image $\left\{D g(d), D g\left(d^{\prime}\right)\right\}$ is degenerate. If there are two such illegal turns then, by choosing each of those two turns (respectively) for the first fold, we get two different Stallings fold factorizations of $g$, hence two different fold lines passing through $X$. Now suppose there is only one illegal turn $T_{1}=\left\{d, d^{\prime}\right\}$ such that $\left\{D g(d), D g\left(d^{\prime}\right)\right\}$ is degenerate. Since some other illegal turn exists, there exists a distinct illegal turn $T^{\prime}=\left\{d^{\prime \prime}, d^{\prime \prime \prime}\right\} \neq T_{1}$ which is mapped to $T_{1}$ by $D g$. For the first fold line passing through $X$, one can use a Stallings fold decomposition for $g$ starting with a maximal fold of $T_{1}$. One obtains a second fold line as follows. Start by folding the two initial segments corresponding to $T_{1}$ some nonmaximal amount, producing a fold segment from $X$ to some $X^{\prime}$. Let $s$ denote the direction in $X^{\prime}$ of the folded segment and $S$ the segment in the direction of $s$ that was obtained by the initial fold of $T_{1}$. Now we must consider separate cases: 
(1) $T_{1}$ and $T^{\prime}$ share no common direction;

(2) $T_{1}$ and $T^{\prime}$ share a common direction.

If (1) holds, we continue by folding the initial segments of $T^{\prime}$ mapping to $S$, return to finish maximally folding $T_{1}$, then continue with any Stallings fold decomposition from there. Now assume (2) holds and $T_{1}$ and $T^{\prime}$ share a common direction, say $d=d^{\prime \prime \prime}$. Let $d^{\prime \prime}$ denote the image in $X^{\prime}$ of the direction of $X$ of the same name. Next fold two initial segments corresponding to the turn $\left\{s, d^{\prime \prime}\right\}$. Again one can continue and obtain a different Stallings fold decomposition, hence a different periodic line passing through $X$.

To obtain two distinct periodic fold lines passing through $\Gamma$, we create periodic fold lines for $\varphi^{2}$ by folding along the Stallings fold decomposition for $f$ from $\Gamma$ to $X$, following one of the distinct fold lines from $X$ to $X$, and then finishing from $X$ to $\Gamma$ the Stallings fold decomposition for $f$.

Another obstacle to an axis bundle having only one axis is the possible existence of multiple affine train track representative on the same point of outer space. The following lemma restricts when this can occur (by Proposition 2.19 each train track representative would induce a distinct $\Lambda$-isometry of the universal cover).

Lemma 4.4 Suppose that $\varphi \in \operatorname{Out}\left(F_{r}\right)$ is ageometric fully irreducible with $i(\varphi)=$ $\frac{3}{2}-r$. Suppose that $X, Y \in \bigcup \operatorname{ST}\left(\varphi^{k}\right)$ and that there exists a $\Lambda$-isometry $\mathcal{I}: \tilde{X} \rightarrow \tilde{Y}$. Then $\mathcal{I}$ is unique.

Proof Suppose there were two distinct $\Lambda$-isometries $\mathcal{I}_{1}, \mathcal{I}_{2}: \tilde{X} \rightarrow \tilde{Y}$. By Lemma 3.9, there is a unique $\Lambda$-isometry $\mathcal{I}_{X}: \tilde{X} \rightarrow T_{+}$and unique $\Lambda$-isometry $\mathcal{I}_{Y}: \tilde{Y} \rightarrow T_{+}$. Let

$$
\mathcal{I}_{X, 1}=\mathcal{I}_{Y} \circ \mathcal{I}_{1}: \tilde{X} \rightarrow T_{+} \quad \text { and } \quad \mathcal{I}_{X, 2}=\mathcal{I}_{Y} \circ \mathcal{I}_{2}: \tilde{X} \rightarrow T_{+} .
$$

Let $x \in \tilde{X}$ be such that $\mathcal{I}_{1}(x) \neq \mathcal{I}_{2}(x)$. Let $L$ be a leaf of $\Lambda$ realized in $\tilde{X}$ and passing through $x$. Since $\mathcal{I}_{1}$ and $\mathcal{I}_{2}$ are $\Lambda$-isometries, $\mathcal{I}_{1}(L)=\mathcal{I}_{2}(L)$. Thus, for each $y \in L$, we have that $\mathcal{I}_{1}(y)$ is a shift of $\mathcal{I}_{2}(y)$ along $L$ by the same distance as $\mathcal{I}_{1}(x)$ is shifted from $\mathcal{I}_{2}(y)$. Since $\mathcal{I}_{Y}$ is a $\Lambda$-isometry, this also holds for $\mathcal{I}_{X, 1}$ and $\mathcal{I}_{X, 2}$. This contradicts the uniqueness of $\mathcal{I}_{X}$.

Lemma 4.5 Suppose that $\varphi \in \operatorname{Out}\left(F_{r}\right)$ is ageometric fully irreducible such that $i(\varphi)=\frac{3}{2}-r$. Then $\mathcal{I} \mathcal{W}(\varphi)$ has no cut vertices if and only if $\mathcal{S A}_{\varphi}=\mathcal{A}_{\varphi}$.

Proof Suppose no component of $\mathcal{I W}(\varphi)$ has a cut vertex. Then, by [14, Lemma 3.1], no train track representative of $\varphi$ has a PNP. That is, every representative of $\varphi$ is fully 
stable. Since $\mathcal{I} \mathcal{W}(\varphi)=\mathcal{I} \mathcal{W}\left(\varphi^{k}\right)$ for all $k \geq 1$, the same can be said for each $\varphi^{k}$ with $k \geq 1$. Thus, $\operatorname{TT}\left(\varphi^{k}\right)=\operatorname{ST}\left(\varphi^{k}\right)$ for each $k \geq 1$ and $\mathcal{S A}_{\varphi}=\mathcal{A}_{\varphi}$, as desired.

Suppose $\operatorname{I} \mathcal{W}(\varphi)$ has a component with a cut vertex. We claim that [14, Lemma 4.3] implies that some power $\varphi^{k}$ has a train track representative (which we can consider to be affine) $g: \Gamma \rightarrow \Gamma$ with a PNP. In order to apply [14, Lemma 4.3], we need to show that there exists a PNP-free train track representative $g^{\prime}: \Gamma^{\prime} \rightarrow \Gamma^{\prime}$ of this sufficiently high power of $\varphi$ satisfying (1)-(3) of the lemma. We take a high enough power $k$ so that the image of every nonperiodic vertex is a fixed vertex and, further, the image of every nonperiodic direction is a fixed direction. We write this representative $g^{\prime \prime}: \Gamma^{\prime \prime} \rightarrow \Gamma^{\prime \prime}$. Since $g^{\prime \prime}$ has no PNP and the ideal Whitehead graph has a component with a cut vertex, there exists a principal vertex $w$ of $\Gamma^{\prime \prime}$ such that $\mathcal{S W}\left(g^{\prime \prime}, w\right)$ has a cut vertex. At the vertex $w$, we fold each gate at $w$ a small amount so that each direction at $w$ is fixed by our new train track representative $g^{\prime}: \Gamma^{\prime} \rightarrow \Gamma^{\prime}$. Now

$$
\mathcal{S W}\left(g^{\prime \prime}, w\right) \cong \mathcal{S} \mathcal{W}\left(g^{\prime}, w\right) \cong \mathcal{L} \mathcal{W}\left(g^{\prime}, w\right),
$$

so that $\mathcal{L} \mathcal{W}\left(g^{\prime}, w\right)$ has a cut vertex, which we will call $x$. We therefore have a decomposition into nontrivial subgraphs $\mathcal{L W}\left(g^{\prime}, w\right)=X_{1} \cup X_{2}$ such that $X_{1} \cap X_{2}=\{x\}$, which verifies (1). Since $g^{\prime}$ is obtained from $g^{\prime \prime}$ by folding at illegal turns, $g^{\prime}$ also has no PNPs. For (2), we need that if $g^{\prime}(v)=w$ for some vertex $v \neq w$, then $\operatorname{Dg}\left(\mathcal{L W}\left(g^{\prime}, v\right)\right)$ is contained in either $X_{1}$ or $X_{2}$. By the proof of Lemma 3.6, in order to have $i(\varphi)=\frac{3}{2}-r$, we need that GI $=\frac{3}{2}-r$, which can only occur when each preperiodic vertex either has valence 2 or has valence 3 and the unique illegal turn. If $v$ has valence 2 , then $\mathcal{L W}\left(g^{\prime}, v\right)$ is a single edge, so its image is in the component. Also in the case where $v$ has valence 3, and the unique illegal turn, the image of $\mathcal{L W}\left(g^{\prime}, v\right)$ is a single edge. In either case, the single edge must lie in either $X_{1}$ or $X_{2}$, which completes the verification of (2). Since $\mathcal{S W}\left(g^{\prime}, w\right)=\mathcal{L W}\left(g^{\prime}, w\right)$ and, in particular, all directions at $w$ are fixed by $D g^{\prime}$, we have that $D g^{\prime}$ is the identity on $\mathcal{L W}\left(g^{\prime}, w\right)$, so that (3) holds. Since $g^{\prime}\left(w^{\prime}\right)=w^{\prime}$, we then have from [14, Lemma 4.3] the desired train track representative $g$ of $\varphi^{k}$ with a PNP.

This $\Gamma$ on which $g$ is a train track map, together with its marking and metric, gives a point in $\mathcal{A}_{\varphi}$. By Lemma 3.9, there cannot additionally be a stable train track representative on that point. Thus, the point is not in $\bigcup \operatorname{ST}\left(\varphi^{k}\right)$. By Proposition 3.11, $\mathcal{S A}_{\varphi}$ is actually the set of stable weak train tracks. And, hence, since a stable weak train track cannot be induced by a train track map unless it is a stable train track map (see Lemma 3.2), $g$ cannot be in $\mathcal{S A}_{\varphi}$.

Theorem 4.6 Suppose $\varphi \in \operatorname{Out}\left(F_{r}\right)$ is ageometric fully irreducible. Then the stable axis bundle $\mathcal{S A}_{\varphi}$ is a unique axis if and only if the rotationless index satisfies $i(\varphi)=$ $\frac{3}{2}-r$. In that case it is a unique periodic fold line. 
Proof Notice that $\mathcal{S A}_{\varphi}$ would have to contain the entirety of the periodic fold line for each affine train track representative on each element of $\bigcup \operatorname{ST}\left(\varphi^{k}\right)$. Suppose that $\mathcal{S A}_{\varphi}$ contained more than one fold line. Since $\bigcup \mathrm{ST}\left(\varphi^{k}\right)$ is dense in $\mathcal{S A}_{\varphi}$, then $\mathcal{S A}_{\varphi}$ would contain stable train tracks on distinct periodic fold lines. Hence, $\mathcal{S} \mathcal{A}_{\varphi}$ would contain distinct periodic fold lines.

First suppose $i(\varphi)>\frac{3}{2}-r$. Then each point in $\bigcup \operatorname{ST}\left(\varphi^{k}\right)$ is contained in at least two distinct periodic fold lines by Lemma 4.3. We thus suppose instead that $i(\varphi)=\frac{3}{2}-r$. It suffices to prove that $\mathcal{S} \mathcal{A}_{\varphi}$ contains a unique periodic fold line.

Before proceeding with the proof, we remark that, for each $T \in \bigcup \operatorname{ST}\left(\varphi^{k}\right)$, there is only one way to fold from $T$ to $T_{+}$. This is because there is only one $\Lambda$-isometry from $T$ to $T_{+}$(by Lemma 3.9) and only one illegal turn (by Lemma 3.6).

We remark further that, for a given $T \in \operatorname{SWTT}(\varphi)$, the realization of $\mathcal{I} \mathcal{W}(\varphi)$ in $T$ is the disjoint union of the stable Whitehead graphs $\mathcal{S W}(w ; T)$ for the principal points $w$ of $T$. Thus, for any $T, T^{\prime} \in \operatorname{SWTT}(\varphi)$ with respective $\Lambda$-isometries $i_{T}: T \rightarrow T_{+}$ and $i_{T^{\prime}}: T^{\prime} \rightarrow T_{+}$, by Lemma 2.36, we have that $i_{T}$ and $i_{T^{\prime}}$ both split minimally (and, in particular, split as much as each other). This allows us to apply Proposition 2.37. That is, for any $T \in \operatorname{SWTT}(\varphi)$, there exists an $\varepsilon>0$ such that, for any $T^{\prime} \in \operatorname{SWTT}(\varphi)$ with $\operatorname{Len}\left(T^{\prime}\right) \leq \varepsilon$, the $\Lambda$-isometry $i_{T}: T \rightarrow T_{+}$factors (uniquely) as a $\Lambda$-isometry $i_{T}: T \rightarrow T^{\prime}$ followed by the $\Lambda$-isometry $i_{T^{\prime}}: T^{\prime} \rightarrow T_{+}$. Since there can only be one fold line from $T^{\prime}$ to $T_{+}$, this implies that $T^{\prime}$ lies on the unique fold line from $T$ to $T_{+}$.

For the sake of contradiction suppose $\mathcal{S} \mathcal{A}_{\varphi}$ contained two distinct periodic fold lines $L$ and $L^{\prime}$. Choose $T \in \bigcup \operatorname{ST}\left(\varphi^{k}\right)$ on $L$, choose $\varepsilon$ as in Proposition 2.37, and then choose $T^{\prime} \in \bigcup \operatorname{ST}\left(\varphi^{k}\right)$ on $L^{\prime}$ so that $\operatorname{Len}\left(T^{\prime}\right)<\varepsilon$. Since the lines converge to $T_{+}$(and $\cup \operatorname{ST}\left(\varphi^{k}\right)$ is dense in $\mathcal{S} \mathcal{A}_{\varphi}$ ), this is possible. Then, by Proposition 2.37, as explained in the previous paragraph, $T$ and $T^{\prime}$ must be on a common fold line. Without generality loss assume that, on the fold line, the parameter of $T$ is less than the parameter of $T^{\prime}$. Thus, for the parameter $t_{0}$ for $T^{\prime}$, we have $L(t)=L^{\prime}(t)$ for all $t \geq t_{0}$.

We claim that there cannot exist two distinct periodic fold lines reparametrizable so that, for some $t_{0}, L(t)=L^{\prime}(t)$ for all $t \geq t_{0}$. Suppose that $L$ is a periodic fold line for $\varphi^{k}$ and $L^{\prime}$ is a periodic fold line for $\varphi^{k^{\prime}}$. Letting $m=k k^{\prime}$, it follows that $L, L^{\prime}$ are both periodic fold lines $\varphi^{m}$. This means that

$$
\varphi^{m \alpha}(L(t))=L(t+m \alpha \log (\lambda)) \quad \text { and } \quad \varphi^{m \alpha}\left(L^{\prime}(t)\right)=L^{\prime}(t+m \alpha \log (\lambda))
$$

for all $t \in \mathbb{R}$ and $\alpha \in \mathbb{Z}$. Given $t \in \mathbb{R}$, this gives us $L(t)=L\left(t^{\prime}\right)$ by using a suitable choice of $t^{\prime} \geq t_{0}$ and $\alpha \in \mathbb{Z}$.

Theorem 4.7 The axis bundle of an ageometric, fully irreducible $\varphi \in \operatorname{Out}\left(F_{r}\right)$ is a unique axis precisely if both of the following two conditions hold: 
(1) the rotationless index satisfies $i(\varphi)=\frac{3}{2}-r$, and

(2) no component of the ideal Whitehead graph $\operatorname{IW}(\varphi)$ has a cut vertex.

Proof If both conditions hold, $\mathcal{A}_{\varphi}$ is a unique axis by Lemma 4.5 and Theorem 4.6.

Suppose $\varphi \in \operatorname{Out}\left(F_{r}\right)$ is ageometric fully irreducible and that $\mathcal{A}_{\varphi}$ is a unique axis. Since $\varphi$ is ageometric, as shown in the opening passage of Section 2.9, $i(\varphi) \geq \frac{3}{2}-r$. If $i(\varphi)>\frac{3}{2}-r$, then each point in $\bigcup \operatorname{ST}\left(\varphi^{k}\right)$ is contained in at least two distinct periodic fold lines by Lemma 4.3. Since each $\varphi$ has a stable train track representative, $\bigcup \operatorname{ST}\left(\varphi^{k}\right)$ is nonempty. $\mathcal{A}_{\varphi}$ would then contain multiple fold lines. So $i(\varphi)=\frac{3}{2}-r$.

The second condition now follows by Lemma 4.5 .

\section{Final remarks}

With the Coulbois computer package [6], the Full Irreducibility Criterion of Pfaff [20, Proposition 4.1], and full irreducibility decidability algorithm of Kapovich [15, Theorem A], it is becoming increasingly easy to check that an outer automorphism satisfies the conditions to have a single-axis axis bundle. Once one determines that it does, they can compute a train track representative using the Coulbois computer package. A Stallings fold decomposition of the representative then gives the periodic line, which is the entire axis bundle.

Ideal decomposition diagrams Let $\varphi \in \operatorname{Out}\left(F_{r}\right)$ be ageometric fully irreducible. Suppose that, in addition to the conditions of Theorem 4.7, the ideal Whitehead graph is connected. Then $\mathcal{A}_{\varphi}$ is still a single periodic fold line. By [19, Proposition 4.3], a power of $\varphi$ has a representative with a Stallings fold decomposition that is a sequence of proper full folds of roses. Also by [19, Proposition 8.3] we know that this decomposition has a realization as a loop in the "ideal decomposition diagram" $\mathcal{I D}(\mathcal{I} \mathcal{W}(\varphi))$. In other words, the single axis in $\mathcal{A}_{\varphi}$ can be viewed as a repeated gradual folding of a loop in $\operatorname{ID}(\mathcal{I} \mathcal{W}(\varphi))$. And this is true for any fully irreducible $\psi$ with $\operatorname{IW}(\psi) \cong \mathcal{I} \mathcal{W}(\varphi)$.

The conjugacy problem One can observe that $\mathcal{A}_{\varphi}$ and $\mathcal{A}_{\psi}$ differ by the action of $\operatorname{Out}\left(F_{r}\right)$ on $\mathrm{CV}_{r}$ if and only if there exist integers $k, l \geq 1$ such that $\varphi^{k}$ and $\psi^{l}$ are conjugate in $\operatorname{Out}\left(F_{r}\right)$. Thus, given two outer automorphisms $\varphi$ and $\psi$ that one has checked, as above, satisfy the conditions for a single axis, one can construct the axis of each to determine if some $\varphi^{k}$ and $\psi^{l}$ are actually conjugate. In fact, $\varphi^{k}$ and $\psi^{l}$ are conjugate if they give the same bi-infinite path in the automata defined in [20]. 
Determining all train track representatives for an outer automorphism In general, it is difficult to identify the set of train track representatives for a given outer automorphism. However, under the conditions of Theorem 4.7, once one has the axis bundle, all train track representatives have the same periodic fold line, namely the single axis of the axis bundle.

\section{References}

[1] M Bestvina, M Feighn, Outer limits (1994) Available at http:// andromeda.rutgers. edu/ feighn/papers/outer.pdf

[2] M Bestvina, M Feighn, M Handel, Laminations, trees, and irreducible automorphisms of free groups, Geom. Funct. Anal. 7 (1997) 215-244 MR1445386

[3] M Bestvina, M Feighn, M Handel, The Tits alternative for $\operatorname{Out}\left(F_{n}\right)$, I: Dynamics of exponentially-growing automorphisms, Ann. of Math. 151 (2000) 517-623 MR1765705

[4] M Bestvina, M Handel, Train tracks and automorphisms of free groups, Ann. of Math. 135 (1992) 1-51 MR1147956

[5] M R Bridson, K Vogtmann, Automorphism groups of free groups, surface groups and free abelian groups, from "Problems on mapping class groups and related topics" (B Farb, editor), Proc. Sympos. Pure Math. 74, Amer. Math. Soc., Providence, RI (2006) 301-316 MR2264548

[6] T Coulbois, Free group automorphisms and train-track representative in python/sage (2012-2014) Sage package Available at https://github.com/coulbois/ sage-train-track

[7] M Culler, K Vogtmann, Moduli of graphs and automorphisms of free groups, Invent. Math. 84 (1986) 91-119 MR830040

[8] A Fathi, F Laudenbach, V Poenaru, Travaux de Thurston sur les surfaces, Astérisque 66-67, Soc. Math. France, Paris (1979) MR568308

[9] M Feighn, M Handel, The recognition theorem for $\operatorname{Out}\left(F_{n}\right)$, Groups Geom. Dyn. 5 (2011) 39-106 MR2763779

[10] D Gaboriau, A Jaeger, G Levitt, M Lustig, An index for counting fixed points of automorphisms of free groups, Duke Math. J. 93 (1998) 425-452 MR1626723

[11] D Gaboriau, G Levitt, The rank of actions on $\mathbf{R}$-trees, Ann. Sci. École Norm. Sup. 28 (1995) 549-570 MR1341661

[12] V Guirardel, Ceur et nombre d'intersection pour les actions de groupes sur les arbres, Ann. Sci. École Norm. Sup. 38 (2005) 847-888 MR2216833

[13] M Handel, L Mosher, Parageometric outer automorphisms of free groups, Trans. Amer. Math. Soc. 359 (2007) 3153-3183 MR2299450 
[14] M Handel, L Mosher, Axes in outer space, Mem. Amer. Math. Soc. 1004 (2011) MR2858636

[15] I Kapovich, Algorithmic detectability of iwip automorphisms, Bull. Lond. Math. Soc. 46 (2014) 279-290 MR3194747

[16] I Kapovich, C Pfaff, A train track directed random walk on $\operatorname{Out}\left(F_{r}\right)$, Internat. J. Algebra Comput. 25 (2015) 745-798 MR3384080

[17] G Levitt, La dynamique des pseudogroupes de rotations, Invent. Math. 113 (1993) 633-670 MR1231840

[18] G Levitt, M Lustig, Irreducible automorphisms of $F_{n}$ have north-south dynamics on compactified outer space, J. Inst. Math. Jussieu 2 (2003) 59-72 MR1955207

[19] C Pfaff, Ideal Whitehead graphs in $\operatorname{Out}\left(F_{r}\right)$, I: Some unachieved graphs, New York J. Math. 21 (2015) 417-463 MR3368968

[20] C Pfaff, Ideal Whitehead graphs in Out $\left(F_{r}\right)$, II: The complete graph in each rank, J. Homotopy Relat. Struct. 10 (2015) 275-301 MR3348263

[21] C Pfaff, Ideal Whitehead graphs in $\operatorname{Out}\left(F_{r}\right)$, III: Achieved graphs in rank 3, J. Topol. Anal. 8 (2016) 207-242 MR3474074

[22] R Skora, Deformations of length functions in groups, preprint, Columbia University (1989)

[23] J R Stallings, Topology of finite graphs, Invent. Math. 71 (1983) 551-565 MR695906

Department of Mathematics and Computer Science, Rutgers University Newark Newark, NJ 07102, United States

Department of Mathematics, University of California, Santa Barbara South Hall, Room 6607, Santa Barbara, CA 93106, United States mosher@andromeda.rutgers.edu, cpfaff@math.ucsb.edu http://www . andromeda.rutgers . edu/ mosher, http://math.ucsb.edu/ cpfaff/

Received: 1 September $2015 \quad$ Revised: 30 April 2016 